Canadian Journal of Applied Linguistics

Revue canadienne de linguistique appliquée

\title{
Maîtrise des clitiques datifs dans les structures bitransitives en français L2 par des apprenants anglophones : influence de la structure argumentale de la L1
}

\author{
Louisette Emirkanian, Leslie Redmond et Adel Jebali
}

Volume 24, numéro 3, automne 2021

URI : https://id.erudit.org/iderudit/1084809ar

DOI : https://doi.org/10.37213/cjal.2021.26419

Aller au sommaire du numéro

\section{Éditeur(s)}

University of New Brunswick

ISSN

1920-1818 (numérique)

Découvrir la revue

\section{Citer cet article}

Emirkanian, L., Redmond, L. \& Jebali, A. (2021). Maîtrise des clitiques datifs dans les structures bitransitives en français L2 par des apprenants anglophones : influence de la structure argumentale de la L1. Canadian Journal of Applied Linguistics / Revue canadienne de linguistique appliquée, 24(3), 30-60. https://doi.org/10.37213/cjal.2021.26419

\section{Résumé de l'article}

L'objectif de notre étude est de mesurer l'influence de la structure argumentale des verbes de la L1, ainsi que celle du sens des verbes, sur la maîtrise des clitiques datifs en francais L2 par des apprenants anglophones. Nous nous intéressons plus précisément aux structures bitransitives. Si le français et l'anglais partagent la structure V SN SP, l'anglais dispose également de la structure à double objet, $\mathrm{V}$ SN SN, pour un sous-ensemble de verbes. Les résultats de notre expérimentation montrent que la structure argumentale de la L1 se manifeste dans la maîtrise des clitiques datifs du français, surtout dans le cas des verbes qui n'acceptent que la structure à double objet en anglais. De plus, le comportement de nos participants face à un sous-ensemble de verbes qui acceptent en anglais l'alternance dative nous a conduits à mener une seconde expérimentation dont les résultats permettent de constater que le sens du verbe a bien un effet sur la performance de nos sujets, effet qui ne peut cependant pas être expliqué par l'influence de la L1.
Copyright (c) Louisette Emirkanian, Leslie Redmond, Adel Jebali, 2021

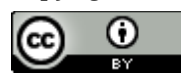

Ce document est protégé par la loi sur le droit d'auteur. L'utilisation des services d'Érudit (y compris la reproduction) est assujettie à sa politique d'utilisation que vous pouvez consulter en ligne.

https://apropos.erudit.org/fr/usagers/politique-dutilisation/ 


\title{
Maîtrise des clitiques datifs dans les structures bitransitives en français L2 par des apprenants anglophones : influence de la structure argumentale de la $\mathrm{L1}^{1}$
}

\author{
Louisette Emirkanian \\ Université du Québec à Montréal \\ Leslie Redmond \\ Memorial University of Newfoundland
}

\author{
Adel Jebali \\ Université Concordia
}

\begin{abstract}
Résumé
L'objectif de notre étude est de mesurer l'influence de la structure argumentale des verbes de la L1, ainsi que celle du sens des verbes, sur la maîtrise des clitiques datifs en français L2 par des apprenants anglophones. Nous nous intéressons plus précisément aux structures bitransitives. Si le français et l'anglais partagent la structure V SN SP, l'anglais dispose également de la structure à double objet, V SN SN, pour un sous-ensemble de verbes. Les résultats de notre expérimentation montrent que la structure argumentale de la L1 se manifeste dans la maîtrise des clitiques datifs du français, surtout dans le cas des verbes qui n'acceptent que la structure à double objet en anglais. De plus, le comportement de nos participants face à un sous-ensemble de verbes qui acceptent en anglais l'alternance dative nous a conduits à mener une seconde expérimentation dont les résultats permettent de constater que le sens du verbe a bien un effet sur la performance de nos sujets, effet qui ne peut cependant pas être expliqué par l'influence de la L1.
\end{abstract}

\begin{abstract}
The objective of this study is to measure the influence of L1 verb argument structure, as well as verb meaning, on the mastery of dative clitics in French as a second language for a group of Anglophone learners. More specifically, we focus on ditransitive structures. While French and English share the V NP PP structure, English also has a double-object structure, V NP NP, for a subset of verbs. The results of our study show that L1 argument structure influences the mastery of dative clitics in French, especially for verbs that only accept the double-object structure in English. Further, the behaviour of our participants with verbs that accept the dative alternation led us to conduct a follow-up study. The findings show that verb meaning also influences performance with dative clitics, but this effect cannot be explained by L1 influence.
\end{abstract}




\section{Maîtrise des clitiques datifs dans les structures bitransitives en français L2 par des apprenants anglophones : influence de la structure argumentale de la L1}

Les recherches en acquisition des langues secondes ont soulevé et bien décrit le phénomène de l'influence translangagière (Odlin, 1989, 2008) que Jarvis et Pavlenko (2008, p. 61) définissent ainsi : « ... the ways in which L2 users' production, perception, and comprehension of forms and structures in one language are affected by their linguistic knowledge of another language. " L'objectif de notre étude est de vérifier si cette influence se manifeste sur le plan de la structure argumentale des verbes bitransitifs dans un contexte particulier, celui où l'argument datif est un clitique.

Nous nous intéressons ici à la réalisation des clitiques datifs du français dans les structures bitransitives du type Marie a donné le livre à Paul-Marie lui a donné le livre. En anglais, la structure Verbe Syntagme Nominal (SN - THĖME) Syntagme Prépositionnel (SP - RÉCIPIENDAIRE) (Mary gave the book to John/to him), qui correspond à celle du français, alterne, pour un sous-ensemble de verbes, avec la structure à double objet, Verbe SN (RÉCIPIENDAIRE) SN (THÈME) (Mary gave John/him the book). Puisque cette alternance dative n'est pas présente en français, la structure bitransitive est particulièrement appropriée pour mesurer, chez les apprenants anglophones du français (L2), l'influence de la L1 dans la réalisation des clitiques datifs.

Dans la plupart des recherches portant sur l'acquisition des clitiques du français, qu'il s'agisse d'acquisition par des enfants ou des adultes, les auteurs (Duffield et coll., 2002; German et coll., 2015; Granfeldt et Schlyter, 2004; Grüter et Crago, 2012; Herschensohn, 2004; Jasmin, 1994; Shimanskaya, 2018; Shimanskaya et Slabakova, 2017; Tsedryk, 2014; Tsedryk et Punko, 2008; White, 1996; pour ne citer que ceux-là) s'entendent en général sur un ordre dans l'appropriation des clitiques par les apprenants du français L2. Ce sont les clitiques datifs et les clitiques génitif et oblique en et $y$ qui sont acquis plus tard que les clitiques accusatifs, les clitiques nominatifs étant maîtrisés les premiers (voir Herschensohn, 2004 et Tsedryk, 2014 pour plus de détails). Certaines études se sont penchées sur le lien entre la structure argumentale et la réalisation des clitiques en français L2 (Shimanskaya, 2018 et Tsedryk, 2014). Shimanskaya a montré que la connaissance qu'ont les apprenants des clitiques accusatifs du français est influencée, au moins en partie, par une non-congruence entre la structure argumentale de leur L1 et celle de leur L2.

L'objectif de notre étude est de vérifier l'impact de la structure argumentale de l'anglais, langue qui possède l'alternance dative pour un sous-ensemble de verbes, sur la réalisation des clitiques datifs en français L2. Nous nous intéressons également à l'incidence du sens du verbe sur la réalisation de ces clitiques dans les structures bitransitives.

\section{L'alternance dative en anglais}

L'alternance dative en anglais a fait l'objet de nombreuses études. Alors que certaines d'entre elles privilégient une approche monosémique (Baker, 1988, en particulier) où les deux variantes sont reliées par dérivation, d'autres optent pour une approche polysémique dans laquelle les deux constructions, V SN SP et V SN SN, sont généralement considérées comme exprimant des sens différents (Green, 1974; Gropen et coll., 1989; 
Krifka, 1999, 2004; Pinker, 1989;). Ainsi, pour Pinker, la phrase en (1a) correspond au sens $(2 a)$, celle en $(1 b)$, au sens $(2 b)$ :

(1) a Ann gave Beth the car.

b Ann gave the car to Beth.

(2) a [EVENT give [Ann Beth [STATE HAVE Beth the car]]] : NP0 CAUSES NP1 to HAVE NP2

b [EVENT give [Ann the car [EVENT GO the car [PATH to [PLACE Beth]]]] : NP0 CAUSES NP2 to GO TO NP1

Dans le premier cas, un état résulte de l'événement (Beth (NP1) possède la voiture (NP2), alors que dans le second, un mouvement sur un chemin possessionnel rend compte du fait que la voiture (NP2) passe de la possession de Ann (NP0) à celle de Beth (NP1). Nous adopterons les termes de Krifka $(1999,2004)$ et nommerons la première structure DO (Double Objet) et la seconde OP (Objet Prépositionnel).

Dans la structure DO, on suppose généralement qu'à la fin de l'événement le récipiendaire possède le thème, ce qui n'est pas nécessairement le cas dans la structure OP (Green, 1974; Krifka, 1999, 2004, entre autres). Ainsi, pour ces auteurs, alors qu'en (3a) on peut supposer que les étudiants ont acquis des connaissances en français, en (3b), en revanche, il est possible qu'ils n'aient rien appris :

(3) a Beth taught the students French. (Exemple (34b) de Krifka, 2004)

b Beth taught French to the students. (Exemple (34a) de Krifka, 2004)

Goldberg (1995), pour qui les constructions sont porteuses de sens, a apporté certaines nuances : le sens central de la construction DO est bien un transfert de possession accompli. Cependant, dans les sens étendus, qu'elle qualifie de métaphoriques, ce transfert de possession réussi n'est pas nécessairement impliqué; c'est le cas par exemple pour des verbes tels que to offer ou to promise.

Dans l'approche lexicaliste de Rappaport Hovav et Levin (2008) et de Levin (2008), le fait que le transfert soit accompli ou non n'est pas lié au type de la structure (DO ou OP), mais au sens du verbe. Pour ces auteures, seuls les verbes du type to give, pour lesquels le récipiendaire ne peut pas être analysé comme le but d'un chemin possessionnel, lexicalisent CAUSE POSSESSION, quelle que soit la structure. Que ces verbes se trouvent donc dans la structure $\mathrm{DO}$ ou dans la structure $\mathrm{OP}$, le récipiendaire possède nécessairement le thème à la fin de l'événement. Par ailleurs, Bresnan et Nikitina (2009) mentionnent dans leur étude la forte tendance à employer la structure SN SN pour le verbe to give, plutôt que la structure SN SP. Elles rapportent que, dans les conversations téléphoniques du Switchboard corpus, ce verbe se trouve dans la construction SN SN dans 84,6 \% des cas. ${ }^{2}$

Parallèlement aux verbes qui acceptent bien les deux structures en anglais, les verbes que nous appellerons OP/DO (to give, to lend, to teach, etc.), il existe deux autres ensembles de verbes, présents dans les structures bitransitives, qui n'acceptent que l'une ou l'autre des formes de l'alternance : d'une part un ensemble de verbes qui n'acceptent que la structure à double objet (verbes DO), par exemple les verbes de non-possession (to refuse, to cost, etc.) comme en (4) où la structure en (b) est non grammaticale; d'autre part, un 
autre ensemble de verbes pour lesquels seule la structure SN SP est grammaticale (verbes OP) (to pull, to donate, to demonstrate, etc.) comme nous le montrent les phrases en (5) :

(4) a The book cost Beth $\$ 40$.

b *The book cost $\$ 40$ to Beth.

(5) a The professor demonstrated the theorem to the students.

b *The professor demonstrated the students the theorem.

Des contraintes d'ordre sémantique et morphophonologique rendent compte de ces différences. ${ }^{3}$

Ces discordances sur le plan de la structure argumentale entre le français et l'anglais ont des répercussions sur le plan de la pronominalisation (Tableau 1). Dans le cas des structures bitransitives en français, le récipiendaire est toujours un SP, et il est pronominalisé par un clitique datif, et non par la forme forte du pronom introduite par la préposition. ${ }^{4}$

\section{Tableau 1}

Structures bitransitives en anglais et en français avec ou sans pronominalisation

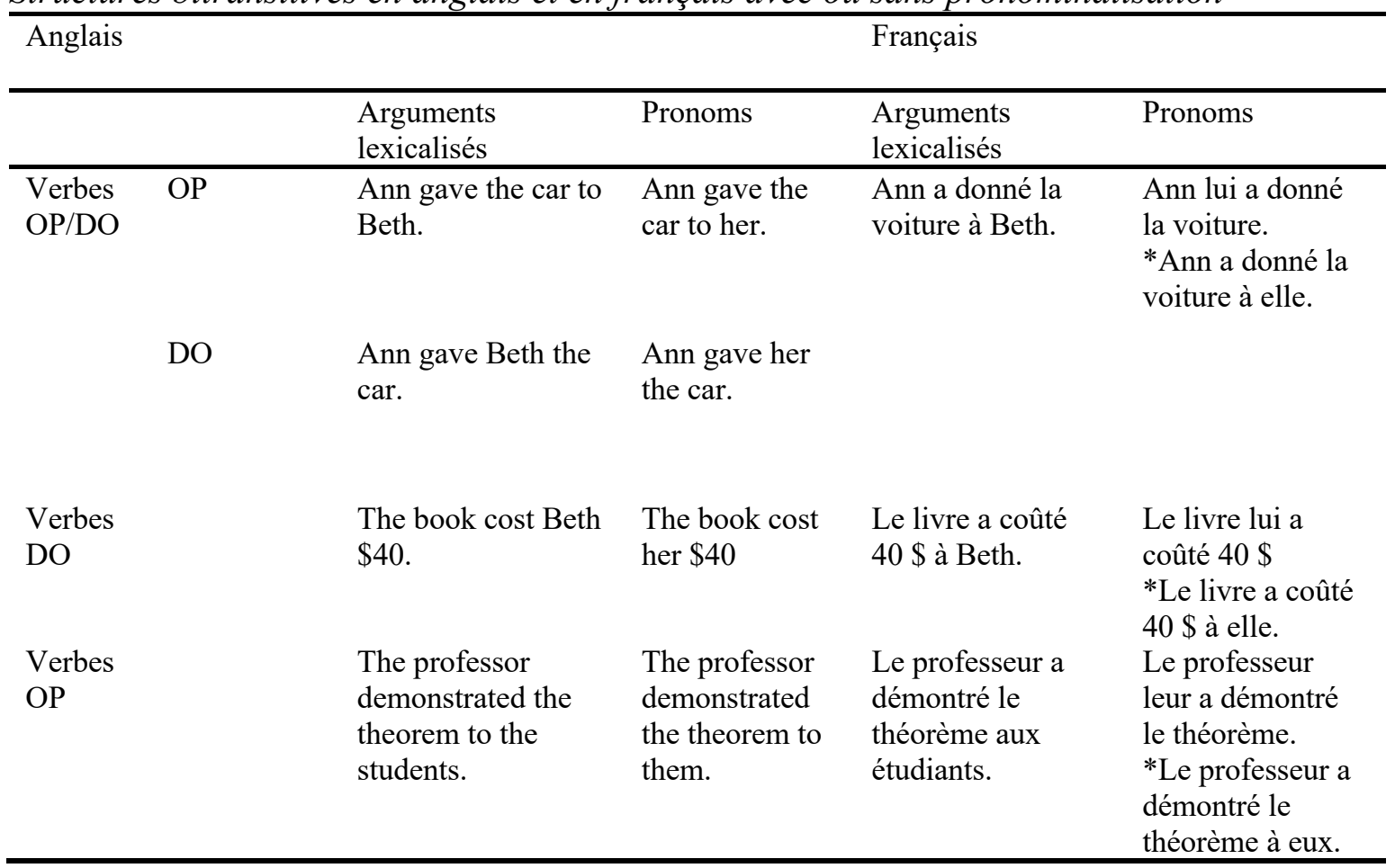

Notre objectif est donc de vérifier si, chez les apprenants anglophones du français L2, la structure argumentale de l'anglais a une incidence sur la réalisation des clitiques datifs présents dans les structures bitransitives du français. De plus, à la lumière des travaux de Goldberg (1995), de Rappaport Hovav et Levin (2008), de Levin (2008) et de Bresnan et Nikitina (2009), il nous a semblé intéressant d'examiner, outre l'influence de la structure argumentale, l'impact du sens des verbes de la classe OP/DO sur la production des clitiques datifs du français. 


\section{L’influence translangagière dans l'acquisition des alternances}

Les recherches sur l'influence translangagière en lien avec la structure argumentale des verbes portent surtout sur les alternances et leur acquisition par des locuteurs dont la langue source est dépourvue de ces alternances ou les exprime différemment de la langue cible. Ces recherches étudient l'impact de la structure argumentale de la L1 sur la performance en L2 en abordant la question sous différents angles, qu'ils soient morphologiques, syntaxiques ou sémantiques. Il ressort de ces recherches que toutes les composantes de la L1, liées aux différents niveaux de représentation linguistique, ne sont pas transférées dans l'interlangue des apprenants, et donc que le transfert est sujet à la modularité. Ces travaux mettent également en relation l'influence de la L1 et le niveau de compétence en L2 : plus l'apprenant est avancé, moins il fait de généralisations sur la base de la structure argumentale de sa L1 (Cabrera et Zubizarreta, 2004; De Souza, 2011; Helms-Park, 2001; Juffs, 1996). Les études de Montrul (2000, 2001), qui portent sur l'acquisition de l'alternance causative/inchoative ${ }^{5}$ et celle des verbes qui dénotent une manière de se déplacer, montrent que globalement, peu importe la L1 des participants, la grammaire universelle peut rendre compte de l'acquisition de certains aspects de ces alternances, l'influence de la L1 se manifestant essentiellement sur le plan morphologique. ${ }^{6}$ Cabrera et Zubizarreta (2004), qui prennent comme point de départ les travaux de Montrul (2000, 2001), étudient plus particulièrement les propriétés constructionnelles de l'alternance ainsi que les propriétés lexicales des verbes qui peuvent subir cette alternance. Elles examinent la manifestation de cette alternance dans l'interlangue d'apprenants de l'anglais et de l'espagnol L2, dont les L1 sont respectivement l'espagnol et l'anglais. Les résultats de leur étude montrent que, selon le niveau de compétence langagière de 1'apprenant, différentes propriétés constructionnelles de la L1 sont transférées dans la L2. Quant aux propriétés lexicales de la L1, elles n'interviennent que chez les apprenants ayant atteint un stade avancé (p. 27).

Outre l'alternance causative, l'alternance dative a également fait l'objet de nombreux travaux de recherche en acquisition des L2. Elle a surtout été étudiée en anglais L2 (Bley-Vroman et Yoshinaga, 1992; Davies, 1994; Inagaki, 1997; Le Compagnon, 1984; Mazurkewich, 1984; McDonough, 2006; Oh, 2010, pour ne citer que ces auteurs) et en espagnol L2 (Bruhn de Garavito, 2006; Cuervo, 2007, par exemple).

L'étude de Cuervo (2007) sur l'acquisition de la structure à double objet en espagnol L2 par des adultes dont la L1 est l'anglais s'inscrit dans le courant des recherches qui prônent que l'on retrouve dans l'interlangue des apprenants d'une L2 les effets de la modularité présents en L1. La structure à double objet est présente en espagnol (Bruhn de Garavito, 2000), comme elle l'est en anglais, mais elle se manifeste de façon différente, la structure DO de l'anglais constituant un sous-ensemble de celle de l'espagnol, le récipiendaire datif en espagnol pouvant être également une source ou un possesseur initial. De plus, la structure DO en espagnol, avec des verbes d'activité ou des verbes d'état, peut exprimer une relation statique. ${ }^{7}$ Cuervo (2007) s'intéresse à la façon dont les sujets de son étude apprennent les propriétés de la DO en espagnol et montre que les propriétés morphosyntaxiques sont acquises avant les propriétés sémantiques, sans toutefois que ce décalage dans l'acquisition de ces propriétés soit relié à l'influence de la L1.

L'étude de Bruhn de Garavito (2006), pour sa part, porte sur les connaissances qu'ont les apprenants de 1'espagnol L2 des restrictions associées au redoublement du 
clitique datif. Elle étudie cette acquisition auprès de locuteurs bilingues (anglais-espagnol), qui ont appris l'espagnol soit de façon précoce (avant l'âge de 6 ans) soit après la puberté, et qu'elle considère comme étant soit des locuteurs natifs soit des apprenants très avancés. Elle a également fait appel à un groupe contrôle de locuteurs monolingues de l'espagnol. Ses résultats montrent qu'il n'y a que très peu de différences entre les trois groupes dans les jugements de grammaticalité. Toutefois, une différence importante se manifeste : le groupe de bilingues tardifs (ceux qui ont appris l'espagnol après l'âge de la puberté) a tendance à restreindre l'emploi du redoublement du clitique datif à des cas où ce clitique possède le trait [+ humain]. Cependant, puisque l'ensemble du groupe ne restreint pas le redoublement à la présence de ce trait, elle nie l'hypothèse selon laquelle cette restriction puisse être due à un transfert de la L1. Qui plus est, les différences entre les différents groupes de bilingues et le groupe de monolingues sont attribuées à des préférences et non à des questions de grammaticalité, les locuteurs natifs préférant fortement le redoublement du clitique à la forme forte (avec préposition). Ces résultats semblent plaider en faveur d'un mécanisme universel à l'œuvre dans l'acquisition des langues secondes.

Qu'en est-il de l'impact de l'alternance dative de l'anglais (L1) sur l'emploi des clitiques datifs du français (L2) ? Tsedryk et Punko (2008) se sont intéressés à l'acquisition du positionnement des clitiques, à celle des propriétés sélectionnelles des verbes ainsi qu'à celle du Cas morphologique par des adultes anglophones. Alors que des différences existent entre les apprenants intermédiaires et avancés sur le plan du positionnement des clitiques et de l'acquisition des propriétés sélectionnelles des verbes, les deux groupes d'apprenants éprouvent la même difficulté à reconnaître les formes casuelles des pronoms. Ainsi, dans cette étude, les apprenants avancés se comportent comme des locuteurs natifs pour ce qui est des propriétés sélectionnelles des verbes. Ces apprenants ont été soumis à un test de jugement de grammaticalité. Tsedryk (2014) s'est penché plus précisément, dans une partie de son étude, sur l'acquisition des clitiques datifs dans les structures bitransitives. La reconnaissance de ces clitiques dans ce type de structures ne semble pas poser de problèmes aux apprenants, ce qui corrobore les résultats de l'étude précédente. Cependant, les résultats de la tâche de production montrent la tendance inverse. Les sujets de l'étude, en effet, éprouvent beaucoup plus de difficultés avec les datifs qu'avec les accusatifs. Tsedryk établit un lien entre les résultats obtenus et l'influence de la structure à double objet de l'anglais. ${ }^{8}$

White s'est intéressée plus directement à l'influence de l'alternance dative de l'anglais en français L2, chez des adultes (White, 1987) et chez de jeunes apprenants (White, 1987, 1991). ${ }^{9}$ Les résultats de son étude de 1987, où elle postule que la forme à double objet est la structure marquée en anglais, montrent que les adultes, tout comme les enfants, acceptent la structure à double objet en français. Les sujets de son étude de 1991, des enfants (âge moyen : 12 ans), soumis à un test de jugement de grammaticalité, ont considéré que les deux formes (SN SN et SN SP) étaient acceptables en français. Ainsi, la L1 correspondant à un surensemble de la L2, l'accord partiel entre les structures de l'anglais et du français induit les apprenants en erreur, la structure argumentale de la L1 étant transférée dans la grammaire de l'interlangue.

Dans le cas de la plupart des alternances, les erreurs commises par les apprenants sont dues à une surgénéralisation, si la L1 possède une alternance que ne possède pas la L2. Dans les cas où la L2 possède une alternance qui n'est pas présente en L1, c'est le phénomène de sous-généralisation qui est mis en œuvre. La tâche de l'apprenant qui sous- 
généralise sera moins difficile, puisqu'il existe dans l'input de la L2 des occurrences de la structure qu'il ne possède pas. En revanche, dans le cas de la surgénéralisation, la tâche sera plus complexe, puisque rien dans l'input de la L2 ne lui permettra d'infirmer l'hypothèse qu'il a faite sur la base des structures de sa L1 (Bruhn de Garavito, 2006; De Souza, 2011; Inagaki, 2001; Montrul, 2001; White, 1991, entre autres). C'est justement ce dernier cas que nous allons examiner : l'alternance dative est présente en anglais, mais pas en français, et cela a des effets sur la réalisation des clitiques datifs dans les structures bitransitives du français.

\section{Objectifs de la recherche et hypothèses}

Notre étude a pour but d'une part de mesurer l'influence de la structure argumentale des verbes de l'anglais sur la production des clitiques datifs du français L2, d'autre part, de vérifier si l'influence de l'anglais se manifeste également sur le plan du sens du verbe, puisque pour certains verbes $\mathrm{OP} / \mathrm{DO}$, ceux de type to give en particulier, la structure DO semble être privilégiée par les locuteurs anglophones (Bresnan et Nikitina, 2009).

Nous postulons que la performance de nos sujets différera selon le type du verbe : $\mathrm{OP}, \mathrm{DO}$ ou OP/DO. Nous pouvons émettre l'hypothèse que les verbes DO, ceux qui ne s'insèrent que dans la structure SN SN en anglais, seront ceux où l'influence de la L1 se manifestera plus souvent et entraîneront davantage d'erreurs, et que les phrases dans lesquelles se trouvent les verbes de type $\mathrm{OP}$, ceux qui ne s'insèrent que dans la structure SN SP, seront au contraire les mieux réussies, puisque cette structure correspond à celle du français. Ainsi, nous supposons que les clitiques datifs seront employés de façon appropriée plus souvent dans les phrases avec des verbes OP que dans celles avec des verbes DO. Nous devrons également vérifier si la structure argumentale de l'anglais a une incidence sur le type d'erreurs commises par les participants.

Pour les verbes qui participent à l'alternance dative, les verbes OP/DO, nous pouvons supposer que, s'il est vrai que la structure DO est associée à un transfert réussi, ce seront les verbes de possession (les verbes de type to give de Rappaport Hovav et Levin, 2008 et de Levin, 2008) qui entraîneront davantage d'erreurs. L'influence de la structure argumentale de l'anglais devrait donc être plus importante pour ces verbes pour lesquels un transfert réussi est inféré que pour les autres verbes de cette classe qui acceptent également les deux structures.

\section{Expérimentation 1}

Notre expérimentation a donc pour objectif d'une part de mesurer l'influence de la structure argumentale de la L1, en l'occurrence l'anglais, sur la production des clitiques datifs en français, d'autre part d'évaluer l'incidence du sens du verbe, dans le cas des verbes qui acceptent les deux structures en anglais, c'est-à-dire les verbes OP/DO. 


\section{Méthode}

\section{Participants}

Cent quatorze étudiants anglophones et allophones (moyenne d'âge : 25,4 ans; 80 femmes et 34 hommes) ont été soumis à un test écrit. Les participants anglophones $(n=92)$ suivaient des cours de français L2 au niveau intermédiaire dans une université anglophone du Canada, lorsque nos données ont été recueillies. Ils avaient tous l'anglais comme L1, parlaient l'anglais à la maison et avaient été scolarisés en anglais au primaire, au secondaire et au postsecondaire. Les étudiants allophones $(n=22)$ étudiaient le français dans les mêmes groupes-cours que les participants anglophones et ils avaient des L1 variées. ${ }^{10}$ Ainsi, constituent-ils un groupe hétérogène.

Différents critères permettent de vérifier l'influence translangagière, l'hétérogénéité intergroupe étant l'un d'eux (Jarvis, 2000; Jarvis et Pavlenko, 2008). ${ }^{11}$ Nous considérons que les allophones constituent un groupe témoin et que, de ce fait, si des différences sont observées entre la performance de nos sujets anglophones et celle de nos sujets allophones, elles pourraient être expliquées par l'influence translangagière.

L'ensemble de nos participants a été soumis à une épreuve constituée de 10 phrases dont le but était de mesurer leur capacité à cliticiser. Dans ces 10 phrases, dans lesquelles le verbe ne sélectionnait qu'un argument interne, les sujets devaient cliticiser un SN accusatif (6 phrases) ou un SP oblique ou génitif (4 phrases); les clitiques le, la, les, l', en ou $y$ étaient donc attendus. Chaque participant a obtenu un score sur 10. Cette variable est traitée comme une variable continue, et elle présente une distribution normale, comme le montre le Tableau 2, puisque les ratios (coefficient/erreur standard) de symétrie et d'aplatissement sont inférieurs à deux (Larson-Hall, 2010, p. 78).

Tableau 2

\begin{tabular}{|c|c|c|c|c|c|}
\hline Moyenne & Écart type & $\begin{array}{l}\text { Indice de } \\
\text { symétrie } \\
\text { (erreur } \\
\text { standard) }\end{array}$ & $\begin{array}{c}\text { Indice } \\
\text { d'aplatissement } \\
\text { (erreur } \\
\text { standard) }\end{array}$ & $\begin{array}{l}\text { Ratio de } \\
\text { symétrie }\end{array}$ & $\begin{array}{c}\text { Ratio } \\
\text { d'aplatissement }\end{array}$ \\
\hline 4,32 & 2,68 & $\begin{array}{c}0,38 \\
(0,226)\end{array}$ & $\begin{array}{l}-0,853 \\
(0,449)\end{array}$ & 1,68 & $-1,90$ \\
\hline
\end{tabular}

Note $: n=114 ; \operatorname{Min}=0 ; \max =10$

En proposant ces 10 phrases à l'ensemble de nos sujets, nous voulions également nous assurer que la capacité à cliticiser de nos participants anglophones était la même que celle de nos participants allophones, puisque l'opérationnalisation de l'influence translangagière repose ici sur l'existence ou non de différences entre les deux groupes de participants. Le résultat d'une analyse de la variance (ANOVA à un facteur) montre que la capacité à cliticiser de chacun des groupes est comparable $[\mathrm{F}(1,113)=0,063, \mathrm{p}=\mathrm{ns}]$.

\section{Procédure et matériel}

La passation du test a été supervisée par l'un des chercheurs, dans la salle de classe. Les sujets disposaient de 45 minutes pour effectuer le test et remplir le questionnaire de 
données sociodémographiques. Le test était constitué de 39 phrases contenant toutes une structure bitransitive. Ces phrases étaient distribuées dans quatre épreuves. Dans les trois premières (31 phrases, exemples (6), (7) et (8)), la tâche consistait à cliticiser des SP datifs, alors que dans la quatrième ( 8 phrases, exemple (9)), il s'agissait de juger de la grammaticalité de phrases du français où les arguments accusatifs et datifs des structures bitransitives étaient lexicalisés.

Les tâches avec cliticisation

Dans l'épreuve 1 (11 items), les sujets devaient cliticiser l'argument datif souligné d'une phrase du français :

(6) Mon étudiant voulait acheter un vélo d'occasion. Comme je ne me servais plus de mon vélo, j'ai vendu mon vélo à mon étudiant.

$\rightarrow$ Comme je ne me servais plus de mon vélo, je lui ai vendu mon vélo.

Dans l'épreuve 2 (8 items), l'épreuve de traduction, ils devaient cliticiser le récipiendaire pronominalisé de la phrase anglaise proposée :

(7) My cousin is back from a trip to Switzerland. Given that his parents have a sweet tooth, he offered chocolates to them.

$\rightarrow$ Il leur a offert des chocolats.

Dans l'épreuve 3 (12 items), les sujets devaient repérer la non-grammaticalité des phrases qui leur étaient proposées. Ils étaient invités à proposer une correction, et donc à utiliser le clitique datif dans la bonne position ou encore à remplacer la forme forte du pronom par un clitique :

Patrick a été engagé en janvier 2010 à Concordia pour enseigner la linguistique aux étudiants de maîtrise. Il a enseigné à eux la syntaxe, la sémantique et la sociolinguistique.

$\rightarrow \square$ Phrase correcte $\quad \square$ Je ne sais pas $\quad \square$ Phrase incorrecte Correction :

Il leur a enseigné la syntaxe, la sémantique et la sociolinguistique.

\section{La tâche sans cliticisation}

Enfin, dans la quatrième épreuve ( 8 items), deux versions d'une phrase en français étaient proposées : l'une grammaticale avec les arguments SN et SP non pronominalisés, l'autre non grammaticale avec deux syntagmes nominaux (l'équivalent de la structure à double objet de l'anglais). Les participants devaient choisir la phrase qu'ils jugeaient grammaticale ou dire qu'à leur avis aucune des deux n'était correcte ou encore que les deux étaient acceptables en français. 
$\square$ Ce livre a coûté Marie $20 \$$.

$\square$ Ce livre a coûté $20 \$$ à Marie.

Les deux phrases sont correctes.

Aucune des phrases n'est correcte.

Que ce soit pour les trois épreuves avec cliticisation ou pour la quatrième sans cliticisation, les trois types de verbes et leurs équivalents français sont présents : les verbes OP ( 9 phrases), les verbes DO (12 phrases) et les verbes OP/DO (18 phrases). ${ }^{12}$ Dans les épreuves 1,3 et 4, les équivalents français des verbes OP sont du type expliquer. Les équivalents français des verbes DO sont du type coûter, essentiellement des verbes de nonpossession. Enfin, les équivalents des verbes OP/DO sont du type donner. ${ }^{13}$ Dans l'épreuve 2, la traduction, les verbes anglais sont les suivants : pour OP, to explain, to demonstrate, pour $\mathrm{DO}$, to cost, to deny, to spare, enfin pour $\mathrm{OP} / \mathrm{DO}$, to offer, to give, to send. Le Tableau 3 présente l'ensemble des verbes utilisés avec leur fréquence en français. ${ }^{14}$

\section{Tableau 3}

Fréquence des verbes utilisés dans l'expérimentation 1

\begin{tabular}{lcc}
\hline Type du verbe en anglais & Verbes & Fréquence des verbes en français $^{15}$ \\
\hline OP & Expliquer (to explain) & 233,92 \\
& Démontrer (to demonstrate) & 12,09 \\
& Annoncer & 133,92 \\
Murmurer & 123,58 \\
DO & Coûter (to cost) & 48,11 \\
& Refuser (to deny) & 152,77 \\
& Épargner (to spare) & 25,99 \\
OP/DO & & 213,99 \\
& Offrir (to offer) & 92,64 \\
& Vendre & 23,04 \\
& Enseigner & 896,01 \\
& Donner (to give) & 159,32 \\
& Apporter & 87,09 \\
& Prêter & 177,64 \\
& Envoyer (to send) & 4832,50 \\
& Dire & 67,44 \\
\hline
\end{tabular}

Afin de contrôler l'effet éventuel de la fréquence des verbes, nous avons vérifié au moyen d'une ANOVA à un facteur qu'il y avait bien absence de lien entre la fréquence des verbes et le type des verbes $[\mathrm{F}(2,15)=1,01, \mathrm{p}=\mathrm{ns}]$.

\section{Codage et analyse des données}

Pour l'ensemble des analyses que nous présentons, nous avons quatre variables indépendantes; deux variables sont liées aux individus, leur L1 et leur capacité à cliticiser; rappelons que cette capacité à cliticiser a été mesurée par la performance des participants aux 10 phrases dans lesquelles le verbe ne sélectionnait qu'un argument interne. Les deux variables reliées au matériel expérimental sont constituées par le type du verbe et le type de la tâche (avec ou sans cliticisation). 
Dans un premier temps, les réponses fournies ont été codées et nous avons d'abord distingué les bonnes réponses des mauvaises, quelle que soit la tâche proposée (avec ou sans cliticisation). Puis nous nous sommes concentrés sur l'analyse des réponses erronées. Trois groupes d'erreurs ont été créés : Datif erroné, Accusatif, Autre réponse. Les réponses Datif erroné sont celles dans lesquelles une forme dative a bel et bien été employée, mais, le plus souvent, avec un positionnement inadéquat (10a), ou avec une erreur morphologique (10b) (emploi du clitique singulier au lieu du pluriel, par exemple) ou encore sous la forme d'un pronom disjoint complément de la préposition (10c) :

(10) a *On a leur enseigné les techniques de la rédaction. / On a enseigné leur les techniques de la rédaction.

b *Il lui démontre l'efficacité de la cafetière. (lui au lieu de leur, dans cet exemple)

c *Il démontre à eux l'efficacité de la cafetière.

Ont été codées Accusatif les réponses dans lesquelles un clitique accusatif est employé à la place du clitique datif, ce clitique accusatif pouvant être bien positionné ou non, et adéquat ou non du point de vue morphologique (11a-b) :

(11) a *Pierre le donne le vieil ordinateur dont il ne se sert plus.

b *Il a la offert un bijou.

La catégorie Autre réponse regroupe les réponses comportant les clitiques en et $y$, celles où les sujets employaient autre chose qu'un pronom, ou ne répondaient à la question que partiellement. ${ }^{16}$

Nous avons eu recours à deux types d'analyses afin de valider statistiquement nos hypothèses. Pour l'analyse de la performance, c'est-à-dire l'opposition entre les bonnes et les mauvaises réponses, nous avons eu recours à des analyses de régression logistique, celles-ci permettant la prédiction d'une réponse donnée exprimée au moyen d'un rapport de chances. Pour l'analyse des erreurs, nous avons eu recours à des analyses de régression logistique multinomiale, puisque notre variable dépendante comprend quatre catégories non ordonnées. Le coefficient de régression s'exprime alors au moyen d'un rapport de risque relatif. L'ensemble de ces analyses a été effectué au moyen du logiciel STATA 13.

\section{Résultats et discussion}

Tout d'abord, nous faisons état de la performance générale de nos deux groupes, les anglophones et les allophones, aux 39 phrases contenant des structures bitransitives et mettons au jour les différences observées. Puis, nous rapportons les résultats de l'analyse des erreurs produites.

\section{Performance générale}

Sur les 3051 réponses obtenues aux 39 phrases pour les deux groupes de participants réunis, ${ }^{17} 1347$ d'entre elles $(44,1 \%)$ sont bien les réponses attendues. Les données descriptives de la Figure 1 présentent la performance générale (pourcentage de bonnes réponses) de chaque groupe, anglophone et allophone, pour chacun des types de 
verbes et pour chacune des deux tâches (avec cliticisation pour les épreuves 1, 2 et 3 et sans cliticisation pour l'épreuve 4).

\section{Figure 1}

Pourcentages de bonnes réponses en fonction du groupe linguistique, du type de verbes et de la tâche

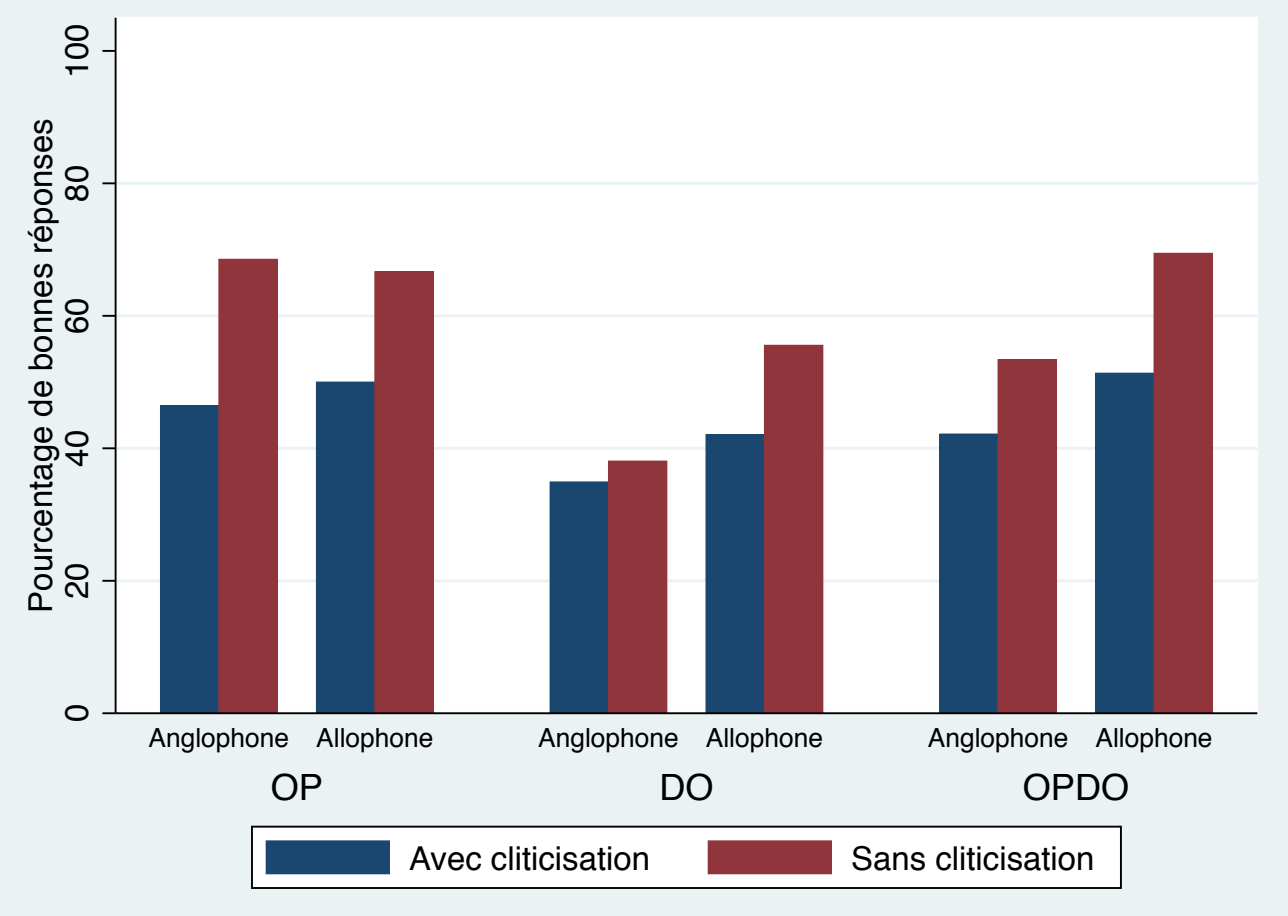

logistique. ${ }^{18}$

Le Tableau 4 contient les rapports de chances issus de l'analyse de régression

\section{Tableau 4}

Régression logistique sur la réussite

\begin{tabular}{lccc}
\hline & Rapport de chances & Erreur type & Sig. \\
\hline Constante & 0,09 & 0,01 & 0,001 \\
$\begin{array}{l}\text { Capacité à cliticiser } \\
\text { Type du verbe }\end{array}$ & 1,39 & 0,02 & 0,001 \\
OP & 1,86 & & \\
- DO (réf.) & 1,63 & 0,21 & 0,001 \\
$\begin{array}{l}\text { OP/DO } \\
\text { - DO (réf.) }\end{array}$ & 1,14 & 0,15 & 0,001 \\
$\begin{array}{l}\text { OP } \\
\text { - OP/DO (réf.) }\end{array}$ & 0,12 & 0,204 \\
$\begin{array}{l}\text { Groupe linguistique } \\
\text {-Anglophone (réf.) }\end{array}$ & 1,31 & 0,13 & 0,007 \\
$\begin{array}{l}\text { Type de la tâche } \\
\text {-Avec cliticisation (réf.) }\end{array}$ & 1,42 & 0,16 & 0,003 \\
\hline Note. Nombre d'observations $=3051 ; \log$ likelihood $=-1829,11 ; \chi^{2}=592,50, \mathrm{p}<0,001 ;$ & $\mathrm{pseudo} \mathrm{R}^{2}=0,1264 ;$
\end{tabular}

réf $=$ catégorie de référence pour les variables catégorielles 
Nous avons effectué une analyse de régression logistique avec quatre effets principaux : la capacité à cliticiser, le type du verbe, le groupe linguistique et le type de la tâche. L'analyse a révélé des effets significatifs pour chacune des variables indépendantes.

Meilleure est leur capacité à cliticiser avec les verbes qui ne sélectionnent qu'un argument interne (accusatif, génitif ou oblique), plus grandes sont les chances de nos sujets de bien cliticiser l'argument datif de la structure bitransitive ou de choisir la phrase grammaticale dans l'épreuve $4(1,39,[\mathrm{p}<0,001])$.

L'effet du type du verbe se manifeste essentiellement dans les oppositions où les verbes DO sont impliqués, verbes qui n'acceptent en anglais que la structure à double objet. En effet, alors que l'opposition OP/DO versus OP n'est pas significative, nos sujets ont 1,86 ( $p<0,001)$ et 1,63 ( $\mathrm{p}<0,001)$ fois plus de chances de réussir les phrases comportant respectivement un verbe OP et un verbe OP/DO que celles avec un verbe DO. Pour ce qui est du type de tâche, nos sujets ont 1,42 fois plus de chances $(p<0,003)$ de produire une bonne réponse dans la tâche sans cliticisation (quatrième épreuve) que dans celle où ils devaient produire un clitique. ${ }^{19}$

Il est particulièrement intéressant de constater que la variable liée au groupe linguistique a un effet, toutes choses étant égales par ailleurs. Les allophones ont en effet 1,31 fois plus de chances $(\mathrm{p}<0,007)$ que les anglophones de fournir une bonne réponse. Rappelons que, pour les 10 phrases qui nous ont permis de mesurer la capacité à cliticiser, il n'y avait pas de différence significative entre les deux groupes. Il s'agit donc d'examiner où se situent les différences pour les phrases bitransitives. Aussi, allons-nous analyser la performance de nos participants par rapport au type du verbe, celle des anglophones d'une part, et celle des allophones d'autre part.

Performance générale - anglophones

Le Tableau 5 fait état de l'analyse de régression logistique pour les participants anglophones.

\section{Tableau 5}

Régression logistique sur la réussite - Anglophones

\begin{tabular}{|c|c|c|c|}
\hline & Rapport de chances & Erreur type & Sig. \\
\hline Constante & 0,10 & 0,01 & 0,001 \\
\hline Capacité à cliticiser & 1,36 & 0,03 & 0,001 \\
\hline \multicolumn{4}{|l|}{ Type du verbe } \\
\hline $\begin{array}{l}\text { OP } \\
\text { - DO (réf.) }\end{array}$ & 1,97 & 0,25 & 0,001 \\
\hline $\begin{array}{l}\text { OP/DO } \\
\text { - DO (réf.) }\end{array}$ & 1,59 & 0,17 & 0,001 \\
\hline $\begin{array}{l}\text { OP } \\
\text { - OP/DO (réf.) }\end{array}$ & 1,24 & 0,14 & 0,06 \\
\hline $\begin{array}{l}\text { Type de la tâche } \\
\text {-Avec cliticisation (réf.) }\end{array}$ & 1,34 & 0,17 & 0,02 \\
\hline
\end{tabular}

Note. . Nombre d'observations $=2445 ; \log$ likelihood $=-1489,87 ; \chi^{2}=354,45, \mathrm{p}<001 ;$ pseudo $\mathrm{R}^{2}=0,1063$; réf $=$ catégorie de référence pour les variables catégorielles 
Nos sujets anglophones ont 1,97 fois plus de chances de fournir une bonne réponse pour les phrases comportant un verbe OP que pour celles avec un verbe DO ( $p<0,001)$. Ils ont 1,59 fois plus de chances de réussir une phrase avec un verbe OP/DO $(\mathrm{p}<0,001)$ qu'avec un verbe DO. L'opposition entre les verbes OP/DO et les verbes OP n'est, quant à elle, pas significative. Nos sujets anglophones éprouvent donc davantage de difficulté avec les verbes qui ne possèdent en anglais que la structure à double objet.

Performance générale - allophones

Le Tableau 6 fait état de l'analyse de régression logistique pour les participants allophones.

\section{Tableau 6}

Régression logistique sur la réussite - Allophones

\begin{tabular}{lccc}
\hline & Rapport de chances & Erreur type & Sig. \\
\hline Constante & 0,13 & 0,03 & 0,001 \\
Capacité à cliticiser & 1,52 & 0,06 & 0,001 \\
$\begin{array}{l}\text { Type du verbe } \\
\text { OP }\end{array}$ & 1,43 & & \\
- DO (réf.) & 1,90 & 0,38 & 0,179 \\
$\begin{array}{l}\text { OP/DO } \\
\text { - DO (réf.) }\end{array}$ & 0,76 & 0,42 & 0,005 \\
$\begin{array}{l}\text { OP } \\
\text { OP/DO (réf.) }\end{array}$ & & 0,19 & 0,256 \\
$\begin{array}{l}\text { Type de la tâche } \\
\text {-Avec cliticisation (réf.) }\end{array}$ & 1,85 & & \\
\end{tabular}

Note. Nombre d'observations $=606 ; \log$ likelihood $=-333,42 ; \chi^{2}=173,08, \mathrm{p}<0,001 ;$ pseudo $\mathrm{R}^{2}=0,2061 ;$ réf $=$ catégorie de référence pour les variables catégorielles

Pour les sujets allophones, contrairement à ce qu'il se passe pour les anglophones, l'opposition OP versus DO n'est pas significative. L'opposition OP/DO versus DO est significative 1,90 $(\mathrm{p}<0,005)$. L'opposition OP/DO versus OP n'est pas significative, tout comme pour les anglophones. recueillies.

Il nous a semblé également intéressant de nous pencher sur les types d'erreurs 


\section{Analyse des erreurs}

Rappelons que nous avons encodé trois types d'erreurs (voir paragraphe Codage et analyse des données) : Datif erroné, Accusatif et Autre réponse. La Figure 2 fait état des données descriptives. ${ }^{20}$

\section{Figure 2}

Pourcentages d'erreurs en fonction du groupe linguistique et du type de verbes

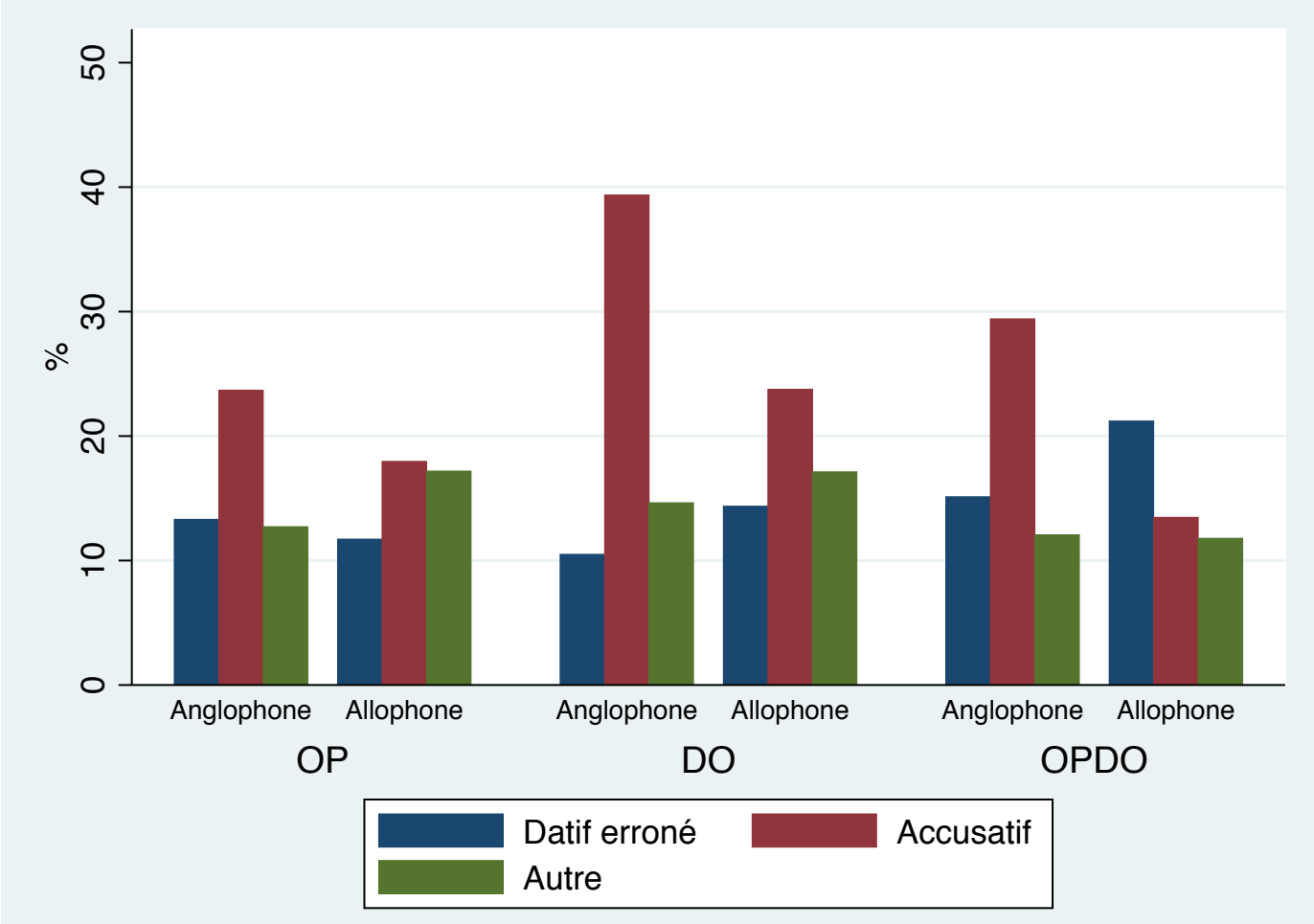

Rappelons que nous avons utilisé une régression logistique multinomiale pour l'analyse des erreurs, la variable dépendante étant catégorielle, composée de quatre modalités non ordonnées. La catégorie de référence de la variable dépendante est toujours bonne réponse dans les rapports de risque relatif que nous présentons dans le Tableau 7. 
Tableau 7

Régression multinomiale sur les erreurs

\begin{tabular}{|c|c|c|c|c|c|c|}
\hline & $\begin{array}{c}\text { Datif } \\
\text { erroné } \\
\text { RRR } \\
\text { (erreur type) }\end{array}$ & Sig. & $\begin{array}{c}\text { Accusatif } \\
\text { RRR } \\
\text { (erreur type) }\end{array}$ & Sig. & $\begin{array}{c}\text { Autre } \\
\text { réponse } \\
\text { RRR } \\
\text { (erreur type) }\end{array}$ & Sig. \\
\hline Constante & $3,83(0,68)$ & 0,001 & $0,10(0,16)$ & 0,98 & $3,06(0,57)$ & 0,001 \\
\hline $\begin{array}{l}\text { Groupe } \\
\text { linguistique } \\
\text {-Allophone } \\
\text { (réf.) }\end{array}$ & $0,82(0,12)$ & 0,152 & $1,97(0,25)$ & 0,001 & $0,94(0,14)$ & 0,67 \\
\hline $\begin{array}{l}\text { Capacité } \\
\text { à cliticiser }\end{array}$ & $0,62(0,02)$ & 0,001 & $0,82(0,02)$ & 0,001 & $0,59(0,02)$ & 0,001 \\
\hline $\begin{array}{l}\text { Type du } \\
\text { verbe } \\
\text { DO } \\
\text { - OP (réf). }\end{array}$ & $1,22(0,22)$ & 0,274 & $2,25(0,29)$ & 0,001 & $1,57(0,27)$ & 0,01 \\
\hline $\begin{array}{l}\text { OP/DO } \\
\text { - OP (réf.) }\end{array}$ & $1,26(0,19)$ & 0,138 & $1,25(0,15)$ & 0,07 & $0,87(0,14)$ & 0,395 \\
\hline $\begin{array}{l}\text { DO } \\
- \text { OP/DO } \\
\text { (réf.) }\end{array}$ & $0,97(0,14)$ & 0,814 & $1,80(0,19)$ & 0,001 & $1,79(0,26)$ & 0,001 \\
\hline
\end{tabular}

Note. Nombre d'observations $=3051 ; \log$ likelihood $=-3460,63, \chi^{2}=786,23, \mathrm{p}<0,001 ;$ pseudo $\mathrm{R}^{2}=0,1020$; réf $=$ catégorie de référence pour les variables catégorielles

La capacité à cliticiser des sujets, le type du verbe et le groupe linguistique ont bien un effet sur certaines des erreurs produites.

Alors que le type du verbe n'a aucune incidence sur la production de l'erreur Datif erroné, il est intéressant de constater qu'il a un effet pour les erreurs Autre réponse et Accusatif, particulièrement dans les oppositions qui mettent en présence les verbes DO. En effet, pour les erreurs Autre réponse, nos sujets, anglophones et allophones réunis, ont 1,57 fois plus de risque de commettre cette erreur avec les verbes DO qu'avec les verbes OP $(\mathrm{p}<0,01)$ et 1,79 fois plus de risque avec les verbes DO qu'avec les verbes OP/DO $(\mathrm{p}<0,001)$. Pour les erreurs liées à l'emploi d'un clitique accusatif, les sujets ont toujours plus de risque de commettre cette erreur pour les verbes DO, qu'on les oppose aux verbes OP ou aux verbes OP/DO. En effet, ils ont 2,25 fois plus de risque d'utiliser un accusatif avec un verbe DO qu'avec un verbe OP $(\mathrm{p}<0,001)$ et 1,80 fois plus de risque avec un verbe DO qu'avec un verbe OP/DO $(\mathrm{p}<0,001)$.

Enfin, qu'en est-il de la variable liée au groupe linguistique? Il est intéressant de noter qu'elle n'est significative que pour l'erreur Accusatif, c'est-à-dire pour le cas où les sujets emploient le clitique accusatif au lieu du clitique datif ou encore acceptent la structure SN SN, plutôt que la structure SN SP dans la tâche sans cliticisation (quatrième épreuve). Les anglophones ont 1,97 fois plus de risque $(\mathrm{p}<0,001)$ que les allophones de fournir comme réponse un élément accusatif. 
Puisque la variable liée au groupe linguistique n'est significative que pour l'erreur Accusatif, nous avons examiné ce que produisent les anglophones et les allophones séparément pour ce type d'erreurs, selon le type du verbe. ${ }^{21}$ Les analyses de régression logistique multinomiale nous montrent que les anglophones ont 2,39 fois plus de risque d'employer un clitique accusatif avec les verbes DO qu'avec les verbes OP $(p<0,001)$. En revanche, cette opposition n'est pas significative pour les allophones.

Nos résultats montrent que la structure argumentale de la L1 a un effet sur l'emploi des clitiques datifs dans les structures bitransitives du français. Chez les sujets anglophones, les phrases comportant des verbes DO (ou leurs équivalents français), ceux qui n'acceptent que la structure SN SN, sont moins bien réussies que les autres. En effet, rappelons que les sujets dont 1'anglais est la $\mathrm{L} 1$ ont 1,97 fois plus de chances de réussir une phrase avec un verbe OP qu'avec un verbe DO. Sur le plan des erreurs, ils ont 2,39 fois plus de risque d'employer un clitique accusatif avec un verbe DO qu'avec un verbe OP. Ainsi, l'influence de l'anglais se manifeste pour les verbes DO, tant sur le plan de la performance générale que sur celui des erreurs. Rappelons que ces oppositions ne sont pas significatives pour les sujets allophones.

Tournons-nous vers les verbes $\mathrm{OP} / \mathrm{DO}$, ceux qui participent à l'alternance dative. Notons d'abord que pour les verbes de cette classe le comportement de nos sujets anglophones ressemble à celui de nos sujets allophones, aussi bien pour l'opposition entre les verbes OP/DO et DO que pour celle entre les verbes OP/DO et OP. Dans la classe des verbes OP/DO figurent, entre autres, des verbes de possession, de type to give, verbes associés à un transfert de possession réussi. Nous avons émis l'hypothèse que si la structure DO est effectivement associée à un transfert nécessairement réussi, les verbes de possession devraient entraîner davantage d'erreurs que les autres verbes également présents dans cette classe OP/DO. Or, nous constatons que c'est le contraire qui se produit. En effet, contrairement à notre hypothèse de départ, nos sujets anglophones ont 1,69 $(\mathrm{p}<0,009)$ plus de chances de réussir les phrases avec un verbe de possession que celles avec d'autres verbes de la classe.

Rappelons que notre hypothèse de départ prenait également en compte les résultats des travaux de Bresnan et Nikitina (2009), qui ont noté une préférence pour la structure SN $\mathrm{SN}$ à l'oral pour le verbe to give en particulier (voir paragraphe $L$ 'alternance dative). Nous avons voulu, dans un premier temps, vérifier si cette préférence se retrouvait à l'écrit, et surtout si elle s'étendait à d'autres verbes de possession. Nous avons donc testé les préférences de locuteurs natifs de l'anglais pour des phrases (9 items) comportant des verbes de possession (to sell, to give, to offer) et les avons opposées à des phrases contenant des verbes de communication (to teach, to write, to read) et de mouvement (to send, to throw, to kick), tous ces verbes acceptant l'alternance dative.

Nous avons effectué un sondage en ligne auprès de sujets canadiens et américains (78 sujets, âge moyen 30,8 ans, 67 femmes et 11 hommes). Les participants devaient indiquer laquelle des structures, SN SN ou SN SP, ils jugeaient la plus naturelle, ou mentionner qu'ils n'avaient vraiment aucune préférence. ${ }^{22}$ Pour les verbes de possession, ceux pour lesquels le transfert est nécessairement accompli, la structure DO est largement préférée à la structure OP. Nos locuteurs anglophones ont choisi 6,03 fois $(p<0,001)$ plus souvent la structure DO pour les verbes de possession que pour les verbes de mouvement et 2,06 fois plus souvent que pour les verbes de communication $(\mathrm{p}<0,007)$. Nos résultats, à l'écrit, corroborent donc ceux de Bresnan et Nikitina (2009). 
Ainsi, alors que les locuteurs natifs de l'anglais semblent bien privilégier la structure DO pour les verbes de possession acceptant l'alternance dative, chez les sujets anglophones soumis à l'expérimentation 1 , les phrases contenant ces verbes ou leurs équivalents français ont donné lieu à davantage de bonnes réponses que les autres phrases.

Afin d'asseoir les résultats obtenus, nous avons choisi de mener une seconde expérimentation portant exclusivement sur les verbes de la classe OP/DO.

\section{Expérimentation 2}

L'objectif de cette seconde expérimentation est donc de vérifier si effectivement les verbes de possession de la classe OP/DO, verbes pourtant associés le plus souvent à la structure SN SN en anglais, sont ceux qui entraînent davantage de bonnes réponses, lorsqu'il s'agit de cliticiser l'argument datif d'une structure bitransitive.

\section{Méthode}

\section{Participants}

Pour cette seconde expérimentation, les réponses de 66 étudiants anglophones (moyenne d'âge : 25,8 ans; 49 femmes et 17 hommes) ont été consignées. Ces participants anglophones suivaient le même cours de français L2 dans une université anglophone du Canada, lorsque nos données ont été recueillies. Ils étaient tous inscrits en $3^{\mathrm{e}}$ année du baccalauréat en études françaises. Ils constituent donc un groupe homogène sur le plan à la fois de leur L1 et de leur maîtrise du français.

\section{Procédure et matériel}

Les sujets disposaient de 30 minutes pour effectuer le test et remplir le questionnaire de données sociodémographiques, sous la supervision d'un des chercheurs.

L'objectif de cette seconde expérimentation étant de mesurer l'influence du sens du verbe, seuls des verbes de type $\mathrm{OP} / \mathrm{DO}$, ceux qui acceptent l'alternance dative en anglais, étaient présents. Ces verbes étaient distribués dans trois épreuves totalisant 18 phrases. Dans deux d'entre elles, la tâche consistait à cliticiser un argument datif (pronominalisation comme en (12), traduction (comme en (13)) et dans l'une d'entre elles, il s'agissait de porter un jugement de grammaticalité sur deux versions d'une phrase, l'une grammaticale, l'autre non grammaticale (comme en (14)).

(12) Au parc, Pierre jouait avec son ami. Il a lancé la balle à son ami. Malheureusement, la balle a atteint l'œil et a causé un hématome.

$\rightarrow$ Il lui a lancé la balle.

(13) Susan's mom sent her a postcard from the Galápagos Islands.

$\rightarrow$ La mère de Susan lui a envoyé une carte postale des îles Galápagos.

(14) $\square$ La secrétaire a envoyé le recueil de notes de cours aux étudiants du département. $\square$ La secrétaire a envoyé les étudiants du département le recueil de notes de cours. $\square$ Les deux phrases sont équivalentes. 
Tout comme pour le sondage en ligne, trois types de verbes de la classe OP/DO sont représentés : des verbes de possession tels que vendre, donner (pour l'épreuve de pronominalisation et pour celle de jugement de grammaticalité), et tels que to sell et to give (pour l'épreuve de traduction), des verbes de communication (transfert cognitif) tels qu'écrire, enseigner, to write, to teach et enfin des verbes de mouvement tels que lancer, envoyer, to throw, to send. L'expérimentation contient 6 items pour chacune des trois épreuves. ${ }^{23}$ Nous avons ici également vérifié, au moyen d'une ANOVA à un facteur, le lien entre les types de verbes et leur fréquence en français. Les résultats confirment l'absence de ce lien $[F(2,5)=0,478, p=n s]$.

\section{Tableau 8}

Fréquence des verbes utilisés dans l'expérimentation 2

\begin{tabular}{|c|c|c|}
\hline Sémantique du verbe & Verbes & Fréquence des verbes en français ${ }^{24}$ \\
\hline \multirow{2}{*}{ Possession } & Vendre & 92,64 \\
\hline & Donner & 896,01 \\
\hline \multirow[t]{2}{*}{ Communication } & Écrire & 305,92 \\
\hline & Enseigner & 23,04 \\
\hline \multirow[t]{2}{*}{ Mouvement } & Lancer & 165,07 \\
\hline & Envoyer & 177,64 \\
\hline
\end{tabular}

\section{Codage et analyse des données}

Le codage et l'analyse des données ont été effectués de la même façon que dans l'expérimentation précédente.

La variable type $d u$ verbe, liée au sens des verbes $\mathrm{OP} / \mathrm{DO}$, est donc à trois modalités : verbes de possession, de communication ou de mouvement. Nous avons également utilisé des analyses de régression logistique pour la validation statistique de nos hypothèses.

\section{Résultats et discussion}

Après avoir rapporté les résultats liés à la performance générale, nous examinons ceux liés aux erreurs.

\section{Performance générale}

Les données descriptives de cette seconde expérimentation sont présentées dans la Figure 3. 


\section{Figure 3}

Pourcentages de bonnes réponses en fonction du type de verbe et de la tâche

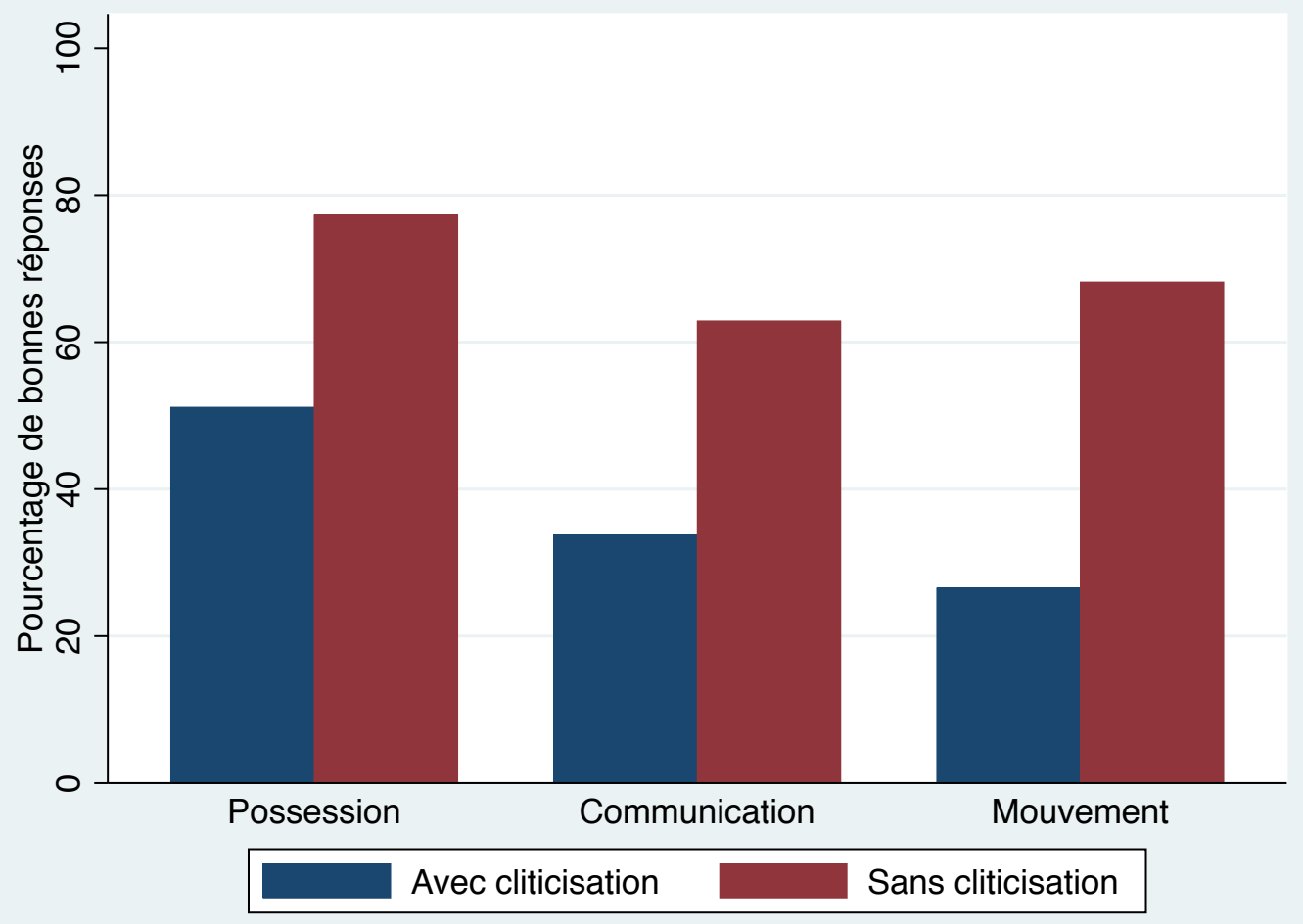

Le Tableau 9 fait état des résultats de l'analyse de régression logistique.

\section{Tableau 9}

Régression logistique sur la réussite (Expérimentation pour les verbes de la classe OP/DO) Rapport de chances $\quad$ Erreur type Sig.

Constante

0,47

0,06

0,001

Type du verbe

Possession

2,08

0,32

0,001

- communication (réf.)

Possession

0,37

0,001

- Mouvement (réf.)

Communication

1,15

0,17

0,362

- Mouvement (réf.)

Type de la tâche

4,03

0,54

0,001

-Avec cliticisation (réf.)

Note. . Nombre d'observations $=1188 ; \log$ likelihood $=-746,89 ; \chi^{2}=151,03, \mathrm{p}<0,001 ;$ pseudo $\mathrm{R}^{2}=0,0918$; réf $=$ catégorie de référence pour les variables catégorielles 
Sur les 1188 réponses obtenues, 569 d'entre elles sont les réponses attendues (47,9\%). Tout comme pour la première expérimentation, les tâches ne demandant pas l'opération de cliticisation sont mieux réussies que celles qui la demandent $(\mathrm{RC}=4,03$, $\mathrm{p}<0,001)$.

Comme on peut le constater, les différences entre les verbes de communication et les verbes de mouvement ne sont pas significatives. Ce sont les phrases dans lesquelles sont présents les verbes de possession qui sont les mieux réussies. En effet, les sujets ont, pour ces verbes où le transfert est nécessairement réalisé, 2,39 fois $(\mathrm{p}<0,001)$ et 2,08 fois plus de chances $(\mathrm{p}<0,001)$ de fournir une bonne réponse que pour les verbes de mouvement et les verbes de communication respectivement. Ces résultats corroborent donc ceux obtenus dans l'expérimentation précédente pour les verbes acceptant l'alternance dative en anglais; nous avions en effet noté que les verbes de possession entraînaient le plus grand nombre de phrases réussies, même si pour ces verbes la structure DO était privilégiée en anglais.

\section{Analyse des erreurs}

La Figure 4 fait état des données descriptives liées aux erreurs.

\section{Figure 4}

Pourcentages de chacun des types d'erreurs en fonction du type de verbes (Expérimentation pour les verbes de la classe OP/DO)

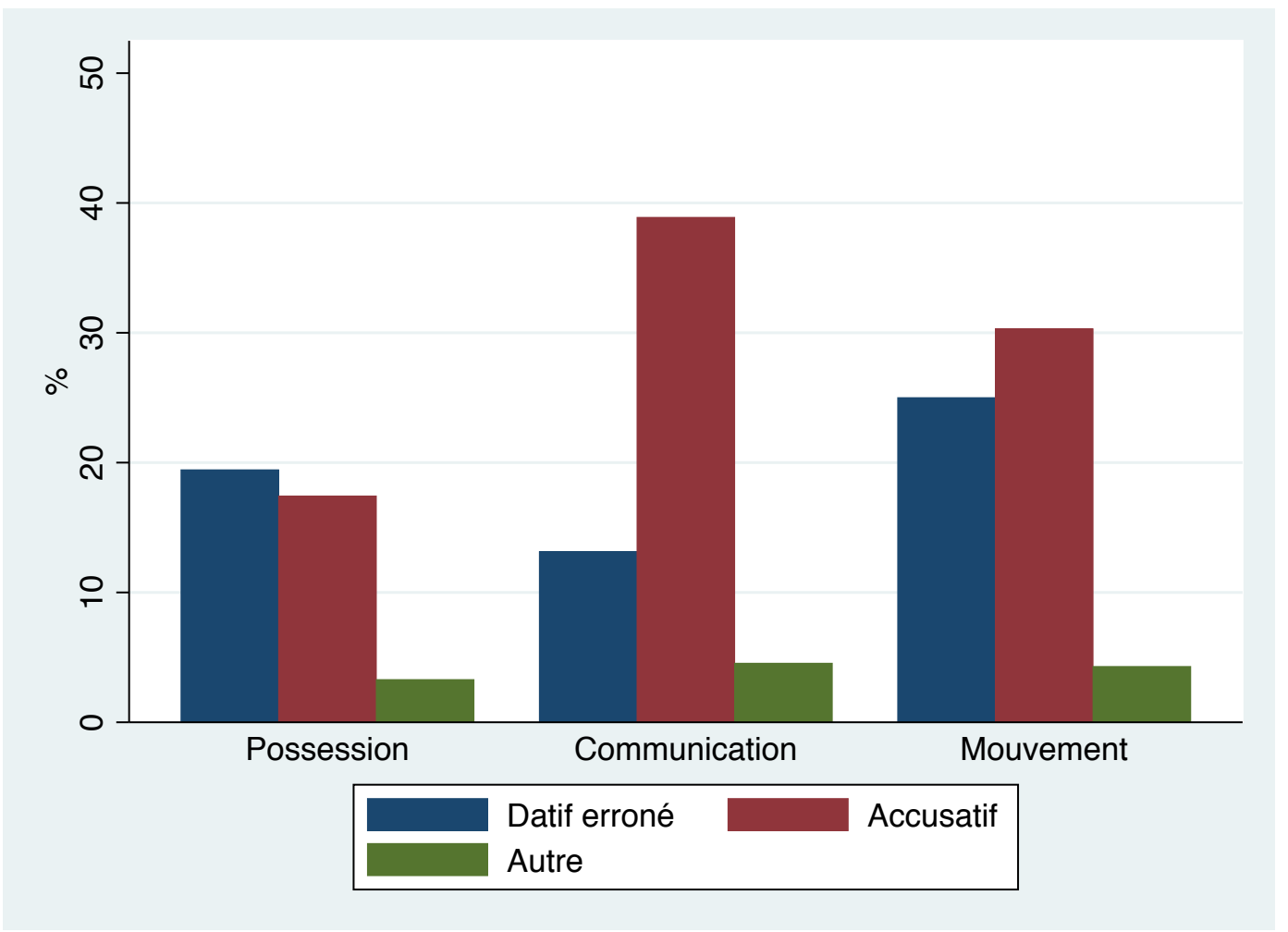


Les résultats de l'analyse statistique sont rapportés dans le Tableau 10.

Tableau 10

Régression multinomiale sur les erreurs (Expérimentation pour les verbes de la classe $O P / D O)$

\begin{tabular}{lcccccc}
\hline & $\begin{array}{c}\text { Datif } \\
\text { erroné } \\
\text { RRR } \\
\text { (erreur } \\
\text { type) }\end{array}$ & Sig. & $\begin{array}{c}\text { Accusatif } \\
\text { RRR } \\
\text { (erreur type) }\end{array}$ & Sig. & $\begin{array}{c}\text { Autre } \\
\text { réponse } \\
\text { RRR } \\
\text { (erreur } \\
\text { type) }\end{array}$ & Sig. \\
\hline $\begin{array}{l}\text { Constante } \\
\text { 0,32(0,04) }\end{array}$ & 0,001 & $0,29(0,04)$ & 0,98 & $0,05(0,02)$ & 0,001 \\
$\begin{array}{l}\text { Type du verbe } \\
\text { Communication- } \\
\text { possession (réf). }\end{array}$ & $0,93(0,19)$ & 0,726 & $3,08(0,54)$ & 0,001 & $1,91(0,72)$ & 0,09 \\
$\begin{array}{l}\text { Mouvement- } \\
\text { possession (réf.) }\end{array}$ & $1,90(0,35)$ & 0,001 & $2,58(0,47)$ & 0,001 & $1,94(0,74)$ & 0,08 \\
$\begin{array}{l}\text { Communication } \\
- \text { Mouvement } \\
\text { (réf.) }\end{array}$ & $0,49(0,10)$ & 0,001 & $1,19(0,20)$ & 0,280 & $0,98(0,35)$ & 0,966 \\
\hline
\end{tabular}

Note. . Nombre d'observations $=1188 ; \log$ likelihood $=-1341,86, \chi^{2}=66,99, \mathrm{p}<0,001 ;$ pseudo $\mathrm{R}^{2}=0,0244$; réf $=$ catégorie de référence pour les variables catégorielles

La régression logistique multinomiale ${ }^{25}$ que nous avons utilisée pour l'analyse des erreurs nous montre que les sujets ont $3,08(\mathrm{p}<0,001)$ fois plus de risque d'employer un clitique accusatif pour les verbes de communication et $2,58(\mathrm{p}<0,001)$ fois plus de risque pour les verbes de mouvement que pour les verbes de possession.

Ainsi, même si pour les verbes de possession la structure SN SN est favorisée en anglais, elle n'influence pas la production des clitiques datifs des structures bitransitives du français. Au contraire, pour ce sous-ensemble de verbes de la classe OP/DO, nos sujets anglophones ont une meilleure performance que pour les autres verbes de la même classe. Ainsi, le sens du verbe a bien un effet sur la performance, qui n'est pas celui auquel nous nous attendions au départ.

\section{Discussion générale}

L'objectif général de cette étude expérimentale était de déterminer si l'influence de la structure argumentale de la L1, l'anglais, se manifeste dans la réalisation des clitiques datifs présents dans les structures bitransitives du français. Dans la première expérimentation, les tâches auxquelles ont été soumis les sujets comportaient trois types de verbes, et leurs équivalents en français : les verbes OP/DO, qui acceptent en anglais l'alternance dative (SN SP / SN SN), les verbes OP, qui n'apparaissent que dans le contexte SN SP, le même qu'en français, les verbes DO, que l'on ne trouve que dans la structure SN SN, absente en français.

Dans les modèles de régression logistique, nous avons constaté que la variable liée au groupe linguistique a un effet. Les anglophones transfèrent la structure SN SN de l'anglais, surtout pour les verbes DO. Les phrases dans lesquelles se trouvent des verbes 
qui n'acceptent en anglais que la structure OP, c'est-à-dire la structure SN SP, la seule présente en français, donnent lieu plus souvent à des clitiques datifs bien réalisés; rappelons que pour le groupe allophone, la différence entre la réussite des clitiques datifs dans les phrases comportant des verbes DO et celle dans les phrases comportant des verbes PO n'est pas significative. Puisque les anglophones (groupe linguistiquement homogène) se comportent de façon différente de celle des allophones (groupe linguistiquement hétérogène), nous postulons que l'influence translangagière chez les anglophones peut expliquer la différence. ${ }^{26}$

Les résultats de la première partie de notre expérimentation rejoignent donc ceux d'autres études (Inagaki, 2001; Montrul, 2001; White, 1991, entre autres), dans lesquelles les chercheurs notent une influence de la structure argumentale de la L1, notamment dans les cas où la L1 correspond à un surensemble de la L2. Cette influence de la L1 se manifeste non seulement dans la performance générale de nos sujets anglophones, mais également dans les différents types d'erreurs qu'ils produisent. En effet, sur le plan des erreurs, la variable liée au groupe linguistique est également significative, en particulier pour l'emploi des accusatifs. Les anglophones emploient davantage de clitiques accusatifs que les allophones. Ces derniers produisent autant de datifs erronés que d'accusatifs (17,2\% de datifs erronés et $17,5 \%$ d'accusatifs pour l'ensemble des verbes). Par ailleurs, nous avons vu que le type du verbe avait un effet sur le type d'erreurs produites, sauf pour l'erreur Datif erroné. Les anglophones ont effectivement plus de risque de commettre une erreur de type Accusatif ou une erreur de type Autre réponse avec les phrases contenant un verbe DO plutôt qu'avec celles dont le verbe est de la classe OP, ce qui n'est pas le cas des allophones. Il y a donc bien un lien entre les clitiques accusatifs produits par les anglophones et la structure argumentale SN SN.

On pourrait cependant avancer que, lorsque les sujets anglophones emploient un datif erroné, il n'y a pas à proprement parler d'influence de la structure argumentale de la L1, mais simplement une difficulté à positionner correctement le pronom ou à l'accorder. Afin d'asseoir nos résultats, nous avons regroupé les bonnes réponses et les datifs erronés de nos sujets anglophones sous la rubrique Datif. L'opposition entre les verbes OP et les verbes DO reste significative. Les sujets anglophones ont 2,09 fois plus de chances de fournir un clitique datif (correct ou erroné) pour les verbes OP que pour les verbes DO $(\mathrm{p}<0,001)$ et 1,74 fois plus de chances $(\mathrm{p}<0,001)$ d'employer un datif avec les verbes $\mathrm{OP} / \mathrm{DO}$ qu'avec les verbes DO.

On pourrait également arguer que la performance des sujets anglophones est liée au fait qu'ils ont de la difficulté à effectuer l'opération de cliticisation, plus particulièrement avec un clitique datif (p. ex. Towell et Hawkins, 1994; Tsedryk et Punko, 2008; Tsedryk, 2014). Rappelons que nous avons pris une mesure de la capacité à cliticiser de nos sujets avec les 10 phrases comportant un seul clitique : les anglophones et les allophones affichaient la même performance. Par ailleurs, nous avons constaté que le type de tâche avait un effet sur la performance et avons noté que l'épreuve sans tâche de cliticisation était mieux réussie que celles avec tâche de cliticisation. Effectivement, nos sujets anglophones ont 1,34 fois plus de chances de fournir une bonne réponse dans l'épreuve sans cliticisation que dans les autres $(\mathrm{p}<0,02)$.

Il est cependant important de noter les deux points suivants. D'une part, s'il est vrai que les phrases avec les verbes OP sont mieux réussies dans la quatrième épreuve que dans les trois premières, il est intéressant d'observer qu'il n'en est pas de même pour celles avec 
des verbes DO. En effet, pour les verbes OP, nos sujets anglophones ont 2,39 fois plus de chances de fournir une bonne réponse dans l'épreuve sans cliticisation que dans les trois autres réunies $(p<0,001)$. En revanche, les rapports de chances ne sont pas significatifs pour les verbes DO, les pourcentages de réussite étant très proches : $38,1 \%$ de bonnes réponses dans l'épreuve sans cliticisation et 34,9\% de bonnes réponses dans celles avec cliticisation. D'autre part, il est intéressant de noter que la différence sur le plan de la performance entre les phrases avec des verbes OP et celles avec des verbes DO est beaucoup plus importante dans l'épreuve sans cliticisation que dans celles où un clitique est attendu. Les sujets ont 3,36 fois plus de chances d'avoir une bonne réponse pour les verbes OP que pour les verbes DO dans cette quatrième épreuve, alors qu'ils ont 1,62 fois plus de chances dans les trois épreuves avec cliticisation ( $p<0,001$ dans les deux cas). Ainsi, même sans avoir à effectuer la tâche de cliticisation, nos sujets anglophones éprouvent toujours de la difficulté avec les phrases comportant un verbe DO.

Il y a donc bien influence de la structure argumentale de la L1, et ce n'est pas le processus plus proprement morphologique qui fait que les phrases comportant des verbes DO, verbes ayant comme arguments internes deux syntagmes nominaux en anglais, sont moins bien réussies.

Nous vérifions donc notre première hypothèse. La structure argumentale de l'anglais a bien une incidence sur la réalisation des clitiques datifs en français L2 dans les structures bitransitives.

En revanche, les résultats des analyses dans lesquelles nous manipulions une variable sémantique ne nous ont pas permis de vérifier notre seconde hypothèse. Ces analyses portaient plus spécifiquement sur les verbes qui acceptent l'alternance dative. Contrairement à ce que nous supposions, les sujets anglophones ont affiché une meilleure performance pour les verbes de possession où le transfert est nécessairement réussi que pour les autres verbes de cette classe OP/DO, alors que les verbes de possession de ce sousensemble favorisent la structure à double objet en anglais. Pour confirmer ce résultat, nous avons soumis des anglophones apprenants du français L2 à une expérimentation ne portant que sur les verbes OP/DO. Les phrases soumises à nos sujets anglophones comportaient des verbes appartenant à trois classes sémantiques : des verbes de communication, de mouvement et de possession.

Les phrases qui affichent le plus haut taux de réussite sont encore une fois celles qui contiennent un verbe de possession. Ainsi nous confirmons les résultats obtenus dans la première expérimentation. Le sens du verbe a bien une incidence sur la performance de nos sujets anglophones, mais elle ne peut être expliquée par l'influence de la L1.

Alors que les verbes de possession favorisent en anglais l'emploi de la structure SN $\mathrm{SN}$, comment expliquer que nos sujets anglophones ont associé à ces verbes plus souvent la structure SN SP, et donc le clitique datif, et ont obtenu un meilleur résultat qu'avec les verbes de communication et de mouvement?

On pourrait avancer que cette bonne performance pour les verbes de possession est en lien avec le fait que ces verbes sont plus fréquents que les verbes de mouvement ou de communication. Or, ce n'est pas le cas; nous avons vérifié qu'il n'y avait pas de lien entre la fréquence des verbes de l'expérimentation et leur appartenance à chacune des catégories (possession, mouvement et communication).

L'explication de cette meilleure performance pour les verbes de possession est cependant bien liée au sens des verbes. En effet, alors que pour les verbes de possession le 
fait que le transfert est réussi constitue une inférence, il n'est qu'une implicature pour les verbes de mouvement, tout comme pour les verbes de communication, qui dénotent des activités (Rappaport Hovav et Levin, 2008). En fait, les verbes de possession du type donner instancient le sens prototypique du transfert de possession et nos sujets anglophones semblent maitriser cette correspondance entre le transfert de possession accompli et la structure SN (THÈME) SP (RÉCIPIENDAIRE) du français. En revanche, ils éprouvent de la difficulté à généraliser l'emploi de ce schéma SN SP aux autres verbes également présents dans les structures bitransitives, qui n'instancient pas un transfert de possession nécessairement réussi.

Plusieurs études ont démontré que les apprenants d'une L2 restreignent leurs hypothèses sur la possibilité de généralisation, sur le plan sémantique, de phénomènes dont ils maîtrisent par ailleurs les propriétés morphosyntaxiques. Par exemple, Bruhn de Garavito (2006), dans son étude portant sur les restrictions associées au redoublement du clitique datif en espagnol L2, constate que les apprenants redoublent le clitique datif de façon adéquate uniquement dans le cas où l'on serait en présence de relations de possession dynamiques. Ce n'est que plus tard qu'ils généralisent le redoublement aux relations de possession statique. Les résultats de l'étude de Cuervo (2007) rejoignent également ceux de l'étude de Bruhn de Garavito (2006). Elle montre que, même s'ils généralisent au-delà des particularités de la construction à double objet de l'anglais, les apprenants anglophones de l'espagnol ne sont pas en mesure de généraliser cette construction jusqu'aux cas instanciant des relations de possession aliénable entre deux entités inanimées. De Souza (2011) observe le même type de phénomène avec l'alternance du mouvement causé pour cinq verbes chez des apprenants lusophones de l'anglais. Il se questionne quant à la capacité des apprenants avancés de généraliser cette alternance à l'ensemble des verbes de l'anglais qui peuvent l'accepter.

Nos apprenants anglophones éprouvent donc la même difficulté à généraliser à l'ensemble des structures bitransitives du français la structure SN SP, qu'ils associent pourtant bien aux verbes de possession.

\section{Conclusion}

L'expérimentation que nous avons menée avait pour objectif d'évaluer la maîtrise, par des sujets anglophones, des clitiques datifs du français présents dans les structures bitransitives et de déterminer si la structure argumentale de l'anglais influait sur la réalisation de ces clitiques. La première partie de l'expérimentation nous a permis de déterminer que la structure argumentale de l'anglais a bien une incidence sur la production des clitiques datifs du français : nos sujets anglophones éprouvent de la difficulté à produire de bonnes réponses surtout dans le cas d'énoncés qui contiennent des verbes n'acceptant en anglais que la structure à double objet (DO). Cette influence de la structure argumentale des verbes se manifeste également dans le cas de la tâche de reconnaissance (jugement de grammaticalité de la quatrième épreuve) où les arguments du verbe ne sont pas pronominalisés. De plus, l'analyse des types d'erreurs nous a permis de constater que le clitique accusatif est plus souvent employé avec les verbes qui n'acceptent que la structure SN SN en anglais. Dans l'expérimentation portant exclusivement sur les verbes $\mathrm{OP} / \mathrm{DO}$, c'est-à-dire ceux qui acceptent l'alternance dative, et où nous manipulions une variable sémantique, nous avons constaté que le sens du verbe a bien un effet sur la performance, et que cet effet n'est pas relié à l'influence de la L1. Alors que les verbes de 
possession, pour lesquels la réussite du transfert est une inférence, favorisent en anglais l'emploi de la structure à double objet, les phrases dans lesquelles ces verbes étaient présents dans notre expérimentation ont donné lieu au plus grand nombre de bonnes réponses avec un clitique datif bien réalisé. Ainsi le lien entre transfert de possession réussi et structure SN (THÈME) SP (RÉCIPIENDAIRE) semble relativement bien acquis pour ces verbes prototypiques par nos sujets; ils ont en revanche plus de difficulté à généraliser l'emploi de cette structure à d'autres verbes présents dans les structures bitransitives. Les résultats de plusieurs études plaident d'ailleurs en faveur du caractère sélectif du transfert, celui-ci se manifestant de manière différente selon l'aspect du phénomène linguistique à l'étude. Montrul (2000) avait justement relevé cette sélectivité du transfert dans son travail sur l'alternance causative.

Nous nous sommes concentrés dans cette étude sur les aspects syntaxiques et sémantiques liés aux structures bitransitives du français. Or, plusieurs des auteurs qui se sont intéressés à l'alternance dative de l'anglais (Bresnan et Nikitina, 2009; Krifka, 1999, 2004; Levin, 2008 et Rappaport Hovav et Levin, 2008, pour ne citer que ceux-là) ont souligné l'importance de la structure informationnelle et celle de la lourdeur des constituants. Ces deux éléments auraient un effet sur le choix des locuteurs, en particulier lorsqu'ils sont en présence d'un verbe qui accepte l'alternance. Par ailleurs, nous avons éliminé de notre recherche les cas où le verbe employé dans la structure bitransitive est un verbe support (donner une claque à quelqu'un, par exemple) ou encore ceux où le verbe et son complément forment une locution (donner du fil à retordre à quelqu'un, par exemple). Il serait intéressant de vérifier l'influence de ces variables sur la production des clitiques datifs en français L2 et nous en tiendrons compte dans la poursuite de notre projet.

La correspondance devrait être adressée à Louisette Emirkanian.

Courriel : emirkanian.louisette@uqam.ca

\section{Notes}

${ }^{1}$ Nous remercions vivement les rédacteurs et les évaluateurs anonymes de la Revue pour leurs commentaires, leurs questions ainsi que leurs recommandations, qui nous ont permis d'améliorer l'article soumis. Nous remercions également Denis Foucambert pour sa disponibilité, les conseils qu'il nous a prodigués tout au long de l'analyse des données ainsi que pour sa lecture attentive de notre article.

${ }^{2}$ Elles mentionnent également que 78,6 \% d'un ensemble de verbes supportant l'alternance sont plutôt employés dans la structure SN SN.

${ }^{3}$ Pour plus de détails sur les contraintes qui régissent l'alternance dative de l'anglais, nous renvoyons le lecteur aux études de Krifka (1999, 2004), de Pinker (1989), de Gropen et coll. (1989) et de Bresnan et Nikitina (2009).

${ }^{4}$ Comme l'a souligné l'un des évaluateurs, précisons que dans le cas des verbes du type penser à, renoncer à, tenir à, c'est bien la préposition suivie de la forme forte du pronom qui est utilisée plutôt que le clitique datif. Cette structure est donc présente dans l'input, mais pas dans le cas des structures bitransitives.

5 Il s'agit de l'alternance suivante (exemples (1a) et (1b) de Montrul (2000, p.234) :

(i) The man broke the window.

(ii) The window broke. 
${ }^{6}$ Dans l'étude de 2000 les L1 et L2 sont l'anglais, l'espagnol et le turc alors que dans celle de 2001 les L1 sont l'espagnol, l'anglais et le turc et les L2, l'espagnol et l'anglais.

${ }^{7}$ En (v), l'argument datif est interprété comme une source. La structure DO exprime une relation statique de possession avec un verbe d'activité en (vi) et un verbe d'état en (vii) (exemples (11), (12a) et (12b) de Cuervo (2007)) :

(v) Emilio le quitó/robó el sombrero a Andreína.

Emilio CLI+datif a pris/volé le chapeau Andreína+datif.

(vi) Emilio le corrigió el informe a Andreína.

Emilio CLI+datif a corrigé le rapport Andreína+datif.

(vii) Emilio le admira la paciencia a Andreína.

Emilio CLI+datif admire la patience Andreína+datif.

${ }^{8}$ L'étude de Tsedryk (2014) ne porte pas directement sur l'influence translangagière. Aussi, n'explore-t-il pas cet aspect du phénomène.

${ }^{9}$ Dans l'article de 1987, White étudie également le cas d'une autre structure marquée (structure à préposition orpheline). Dans l'article de 1991, elle expérimente également le cas où la L1 est un sous-ensemble de la L2, à travers l'étude de l'ordre du verbe et de ses arguments et de ses adjoints.

${ }^{10}$ Nous avons dénombré treize L1 différentes chez nos participants allophones : roumain $(\mathrm{n}=2)$, albanais $(\mathrm{n}=1)$, arabe $(\mathrm{n}=1)$, bulgare $(\mathrm{n}=1)$, chinois $(\mathrm{n}=2)$, espagnol $(\mathrm{n}=6)$, finnois $(\mathrm{n}=2)$, grec $(\mathrm{n}=1)$, farsi $(\mathrm{n}=1)$, portugais $(\mathrm{n}=2)$, russe $(\mathrm{n}=1)$, ukrainien $(\mathrm{n}=1)$, vietnamien $(\mathrm{n}=1)$. Ces sujets suivaient des cours dans différents programmes de baccalauréat de l'université où l'expérimentation a été menée.

${ }^{11}$ On peut confirmer l'hétérogénéité intergroupe «[...] when comparable learners of a common L2 who speak different L1s diverge in their Il [interlanguage] performance [...] » (Jarvis, 2000, p. 254).

${ }^{12}$ Dans la construction des items, nous avons pris en compte la lourdeur des constituants, qui peut favoriser pour les verbes OP/DO le choix l'une ou l'autre des structures. Nous avons veillé, lorsque cela était nécessaire, à employer des arguments ayant le même poids. En revanche, cette variable n'a pas été contrôlée.

${ }^{13}$ Un même verbe peut apparaître dans différents items.

${ }^{14}$ Nous nous sommes fondés sur les données de Lexique.org (http://www.lexique.org).

${ }^{15}$ Puisque la variable n'est pas normalement distribuée, nous avons pris le log 10 des valeurs, ce qui rend la variable normalement distribuée.

${ }^{16}$ Plus précisément, pour l'épreuve 3 (correction des phrases non grammaticales), la réponse Je ne sais pas est incluse dans cette dernière catégorie, de même que les cas où l'étudiant cochait Phrase incorrecte, sans toutefois proposer une correction (voir exemple [8]).

${ }^{17}$ Alors que 4446 réponses étaient attendues, seules 3051 ont été données. Les données manquantes, indépendantes de notre volonté, n'ont pas été analysées.

${ }^{18}$ Les analyses statistiques de régression logistique prennent en compte l'effet de chaque variable de façon indépendante. En effet, les rapports de chances sont vrais toutes choses étant égales par ailleurs.

${ }^{19}$ Nous reviendrons sur l'effet du type de tâche dans la discussion, plus particulièrement pour les sujets anglophones. 
${ }^{20}$ Les pourcentages ont été calculés sur le nombre total d'occurrences incluant les bonnes réponses. Notons que pour l'analyse des erreurs nous ne tenons pas compte de la variable liée au type de tâche.

${ }^{21}$ Nous avons effectué deux analyses de régression logistique multinomiale, l'une pour les sujets anglophones, l'autre pour les sujets allophones. Les valeurs rapportées proviennent de ces analyses.

${ }^{22}$ Les items de cette expérimentation en ligne avaient la forme suivante :

1- Peter gave his grandmother chocolate.

2- Pierre gave chocolate to his grandmother.

A- $\square \quad$ I prefer sentence 1 .

$B-\square \quad$ I prefer sentence 2 .

$C-\square \quad$ Both sentences are the same.

23 Tout comme pour l'expérimentation 1, nous avons pris en compte la lourdeur des constituants, sans toutefois contrôler cette variable. Par ailleurs, pour tous les items, le récipiendaire est [+ANIMÉ].

${ }^{24}$ Puisque la variable n'est pas normalement distribuée, nous avons pris la racine carrée des valeurs, ce qui rend la variable normalement distribuée.

${ }^{25}$ Comme pour l'expérimentation précédente, Bonne réponse est la catégorie de référence de la variable dépendante.

${ }^{26}$ Étant donné la grande variété des L1 de nos sujets allophones et du faible nombre de sujets pour chacune d'elles, nous n'avons pas été en mesure d'établir des liens entre leur performance et leur L1.

\section{Références}

Baker, M. C. (1988). Incorporation: A theory of grammatical function changing. University of Chicago Press.

Bley-Vroman, R. et Yoshiniga, N. (1992). Broad and narrow constraints on the English dative alternation: some fundamental differences between native speakers and foreign language learners. University of Hawai'i Working Papers in ESL, 11, 157-199.

Bresnan, J. et Nikitina, T. (2009). On the gradience of the dative alternation. Dans L. Uyechi et L.H. Wee (dir.) Reality Exploration and Discovery: Pattern Interaction in Language and Life (p. 161-184). Stanford: CSLI Publications.

Bruhn de Garavito, J. (2000). The syntax of Spanish multifunctional clitics and near-native competence (Thèse de doctorat inédite). Université McGill, Montréal.

Bruhn de Garavito, J. (2006). Knowledge of clitic doubling in Spanish: Evidence against pattern learning. Dans R. Slabakova, S. A. Montrul et P. Provost (dir.), Inquiries in linguistic development: In honor of Lydia White (p. 305-333). Amsterdam: John Benjamins.

Cabrera, M. et Zubizarreta, M. L. (2004). Constructional properties versus lexical specific transfer: Overgeneralized causatives in L2 English and L2 Spanish. Boston University Conference on Language Development, 28, Proceedings Supplement, 15-30. 
Cuervo, M. C. (2007). Double objects in Spanish as a second language. Studies in Second Language Acquisition, 29, 583-615. DOI: 10.1017/S0272263107070441

Davies, W. D. (1994). English dative alternation and evidence for a thematic strategy in adult SLA. Issues in Applied Linguistics, 5, 59-82.

De Souza, R. A. (2011). Argument structure in L2 acquisition: Language transfer revisited in a semantics and syntax perspective. Ilha do Desterro, 60, 153-188.

DOI : $10.5007 / 2175-8026$

Duffield, N., White, L., Bruhn De Garavito, J., Montrul, S. et Prévost, P. (2002). Clitic placement in L2 French: Evidence from sentence matching. Linguistics, 38, 487-525.

German, E. S., Herschensohn, J., et Frenck-Mestre, C. (2015). Pronoun processing in anglophone late L2 learners of French: Behavioral and ERP evidence. Journal of Neurolinguistics, 34, 15-40.

Goldberg, A. (1995). Constructions: A construction grammar approach to argument structure. Chicago: University of Chicago Press.

Granfeldt, J. et Schlyter, S. (2004). Cliticisation in the acquisition of French as LI and L2. Dans P. Prévost et J. Paradis (dir.), The acquisition of French in different contexts: Focus on functional categories (p. 333-370). Amsterdam: John Benjamins.

Green, G. (1974). Semantics and syntactic regularity. Bloomington: Indiana University Press.

Gropen, J., Pinker, S., Hollander, M., Goldberg, R. et Wilson, R. (1989). The learnability and acquisition of the dative alternation in English. Language, 65, 203-257.

Grüter, T. et Crago, M. (2012). Object clitics and their omission in child L2 French: The contributions of processing limitations and L1 transfer. Bilingualism: Language and Cognition, 15, 531-549.

Helms-Park, R. (2001). Evidence of lexical transfer in learner syntax. Studies in Second Language Acquisition, 23, 71-102

Herschensohn, J. (2004). Functional categories and the acquisition of object clitics in L2 French. Dans P. Prévost et J. Paradis (dir.), The acquisition of French in different contexts: Focus on functional categories (p. 207-242). Amsterdam: John Benjamins.

Inagaki, S. (1997). Japanese and Chinese learner's acquisition of the narrow-range rules for the dative alternation in English. Language Learning, 47, 637-669.

Inagaki, S. (2001). Motion verbs with goal PPs in the L2 acquisition of English and Japanese, Studies in Second Language Acquisition, 23, 153-170.

Jarvis, S. (2000). Methodological Rigor in the Study of Transfer: Identifying L1 Influence in them Interlanguage Lexicon. Language Learning, 50, 245-309. http://10.1111/0023-8333.00118

Jarvis, S. et Pavlenko, A. (2008). Crosslinguistic Influence in Language and Cognition. Routledge.

Jasmin, L. (1994). Acquisition des pronoms clitiques objets par des apprenants adultes du français langue seconde, [thèse de doctorat, Université d'Ottawa, Ottawa]. http://hdl.handle.net/10393/6743 
Juffs, A. (1996). Semantics-syntax correspondances in second language acquisition. Second Language Research, 12, 177-221.

Krifka, M. (1999). Manner in dative alternation. WCCFL 18, Cascadilla Press.

Krifka, M. (2004). Semantic and pragmatic conditions for the dative alternation. Korean Journal of English Language and Linguistics, 4, 1-32.

Larson-Hall, J. (2010). A guide to doing statistics in second language research using SPSS. Routledge.

Le Compagnon, B. (1984). Interference and overgeneralization in second language learning: The acquisition of English dative verbs by native speakers of French. Language Learning, 34, 39-67.

Levin, B. (2008). Dative verbs. A crosslinguistic perspective. Lingvistica Investigationes, $31,285-312$.

Mazurkewich, I. (1984). The acquisition of the dative alternation by second language learners and linguistic theory. Language Learning, 34, 91-109.

McDonough, K. (2006). Interaction and syntactic priming: English L2 speakers' production of dative constructions. Studies in Second Language Acquisition, 28, 179-207.

Montrul, S. (2000). Transitivity alternations in L2 acquisition. Toward a modular view of transfer. Studies in Second Language Acquisition, 22, 229-273.

Montrul, S. (2001). Agentive verbs of manner of motion in Spanish and English as second languages. Studies in Second Language Acquisition, 23, 171-206.

Odlin, T. (1989). Language transfer: Cross-linguistic influence in language learning. Cambridge University Press.

Odlin, T. (2008). Cross-linguistic influence. Dans C. J. Doughty et M. H. Long (dir.), The handbook of second language acquisition (p. 436-486). Blackwell Publishing.

Oh, E. (2010). Recovery from first-language transfer: The second language acquisition of English double objects by Korean speakers. Second Language Research, 26, 3, 407439.

Pinker, S. (1989). Learnability and cognition, The acquisition of argument structure. MIT Press.

Rappaport Hovav, M. et Levin, B. (2008). The English dative alternation: The case for verb sensitivity. Journal of Linguistics, 44, 129-167.

Shimanskaya, E. (2018). Another look at L2 acquisition of French clitics and strong pronouns. Dans J. Cho, M. Iverson, T. Judy, T. Leal et E. Shimanskaya (dir.), Meaning and Structure in Second Language Acquisition: In honor of Roumyana Slabakova (p. 66-94). John Benjamins.

Shimanskaya, E., \& Slabakova, R. (2017). Re-assembling objects: A new look at the L2 acquisition of pronominal clitics, Bilingualism: Language and Cognition, 20, 512-529. http://doi.org/10.1017/S1366728915000784

Towell, R. et Hawkins, R. (1994). Approaches to second language acquisition. Multilingual Matters.

Tsedryk, K. et Punko, I. (2008). L'acquisition des pronoms clitiques en français langue seconde. In J. Durand et B. Habert, CMLF2008, EDP Sciences http://doi.org/10.1051/cmlf08026. 
Tsedryk, K. (2014). Les interactions morphosyntaxiques dans l'interlangue des apprenants : le cas de l'acquisition des pronoms clitiques en français L2. (Thèse de doctorat, University of Western Ontario). Electronic Thesis and Dissertation Repository (2062). http://ir.lib.uwo.ca/etd/2062

White, L. (1987). Markedness and second language acquisition: The question of transfer. Studies in Second Language Acquisition, 9, 261-286.

White, L. (1991). Argument structure in second language acquisition. French Language Studies, 1, 189-207.

White, L. (1996). Clitics in L2 French. Dans H. Clahsen, (dir.), Generative approaches to first and second language acquisition: Empirical findings, theoretical considerations, and cross-linguistic comparisons (p. 335-368), John Benjamins. 